\title{
Pacific
}

Journal of

Mathematics

\section{A PROOF OF THE CONCUS-FINN CONJECTURE}

KIRK E. LANCASTER 


\title{
A PROOF OF THE CONCUS-FINN CONJECTURE
}

\author{
KIRK E. LANCASTER \\ To Paul Concus and Robert Finn
}

Consider a nonparametric capillary or prescribed mean curvature surface $z=f(x, y)$ defined in a cylinder $\Omega \times \mathbb{R}$ over a two-dimensional region $\Omega$ that has a boundary corner point at $O$ with an opening angle of $2 \alpha$. Suppose $2 \alpha \leq \pi$ and the contact angle approaches limiting values $\gamma_{1}$ and $\gamma_{2}$ in $(0, \pi)$ as $O$ is approached along each side of the opening angle. Our results yield a proof of the Concus-Finn conjecture, which provides the last piece of the puzzle of determining the qualitative behavior of a capillary surface at a convex corner. We find that

- if $\left(\gamma_{1}, \gamma_{2}\right)$ satisfies $2 \alpha+\left|\gamma_{1}-\gamma_{2}\right|>\pi$, then $f$ is bounded but discontinuous at $O$ and has radial limits at $O$ from all directions in $\Omega$ and, these radial limits behave in a prescribed way;

- if $\left(\gamma_{1}, \gamma_{2}\right)$ satisfies $\left|\gamma_{1}+\gamma_{2}-\pi\right|>2 \alpha$, then $f$ is unbounded in every neighborhood of $O$; and

- otherwise $f$ is continuous at $O$.

\section{Introduction and statement of theorems}

Let $\Omega \subset \mathbb{R}^{2}$ be a connected, open set. Consider the prescribed mean curvature problem

$$
\begin{aligned}
N f & =H(\cdot, f(\cdot)) & & \text { in } \Omega, \\
T f \cdot \boldsymbol{v} & =\cos \gamma & & \text { almost everywhere on } \partial \Omega,
\end{aligned}
$$

where $T f=\nabla f / \sqrt{1+|\nabla f|^{2}}, N f=\nabla \cdot T f, \boldsymbol{v}$ is the exterior unit normal on $\partial \Omega$, $H(x, t)$ is a weakly increasing function of $t$ for each $x \in \Omega$ and $\gamma=\gamma(x) \in[0, \pi]$. If (1) specifically is

$$
N f=\kappa f+\lambda \quad \text { in } \Omega
$$

(that is, $H(x, t)=\kappa t+\lambda)$, where $\kappa$ and $\lambda$ are constants with $\kappa \geq 0$, then the surface $z=f(x)$ for $x \in \Omega$ represents the stationary liquid-gas interface formed

MSC2000: primary 76B45; secondary 35J60, 53A10.

Keywords: Concus-Finn conjecture, capillary graph. 


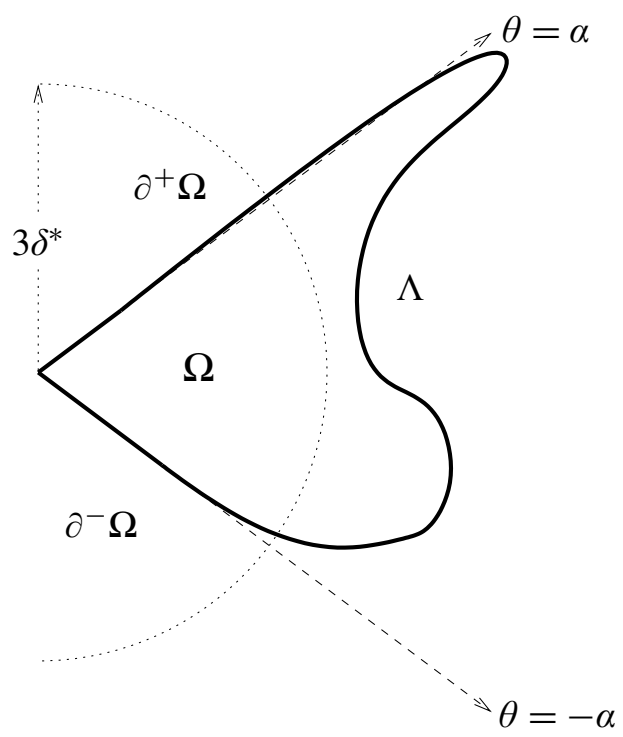

Figure 1. The domain $\Omega$.

by an incompressible fluid in a vertical cylindrical tube with cross section $\Omega$ in a microgravity environment or in a downward oriented gravitational field; here the subgraph $U=\{(x, t) \in \Omega \times \mathbb{R}: t<f(x)\}$ represents the fluid-filled portion of the cylinder and $\gamma(x)$ is the angle at which the liquid-gas interface meets the vertical cylinder at $(x, f(x))$ [Finn 1986].

Since 1970, Paul Concus and Robert Finn have made fundamental contributions to the mathematical theory of capillary surfaces and have discovered that these surfaces can behave in very peculiar and unexpected ways; see for example [Finn $1999 ; 2002 b ; 2002 a]$. Of particular interest, to both the mathematical and physical theories in vertical cylinders, are domains $\Omega$ whose boundaries contain corners.

Suppose $O=(0,0) \in \partial \Omega$ and $\Omega$ has a corner of size $2 \alpha \leq \pi$ at $O$. With $\Omega$ as illustrated in Figure 1, suppose there exist $\gamma_{1}, \gamma_{2} \in(0, \pi)$ such that

$$
\lim _{\partial^{+} \Omega \ni x \rightarrow(0,0)} \gamma(x)=\gamma_{1} \quad \text { and } \quad \lim _{\partial^{-} \Omega \ni x \rightarrow(0,0)} \gamma(x)=\gamma_{2} .
$$

Then Figure 2 can be used to illustrate our knowledge of the behavior of a solution $f$ of (3) and (2) at the corner $O$; here let $R, D_{1}^{ \pm}$and $D_{2}^{ \pm}$be the indicated open regions in the (open) square $(0, \pi) \times(0, \pi)$. If $\left(\gamma_{1}, \gamma_{2}\right) \in \bar{R} \cap(0, \pi) \times(0, \pi)$, then $f$ is continuous at $O$; see [Concus and Finn 1996, Theorem 1; Lancaster and Siegel 1996a, Corollary 4]. If $\left(\gamma_{1}, \gamma_{2}\right) \in D_{1}^{ \pm}$, then $f$ is unbounded in any neighborhood of $O$ and the capillary problem has no solution if $\kappa=0$ [Concus and Finn 1996; Finn 1996]. If $\left(\gamma_{1}, \gamma_{2}\right) \in D_{2}^{ \pm}$, then $f$ is bounded [Lancaster and Siegel 1996a, Proposition 1] but its continuity at $O$ is unknown. Concus and Finn 


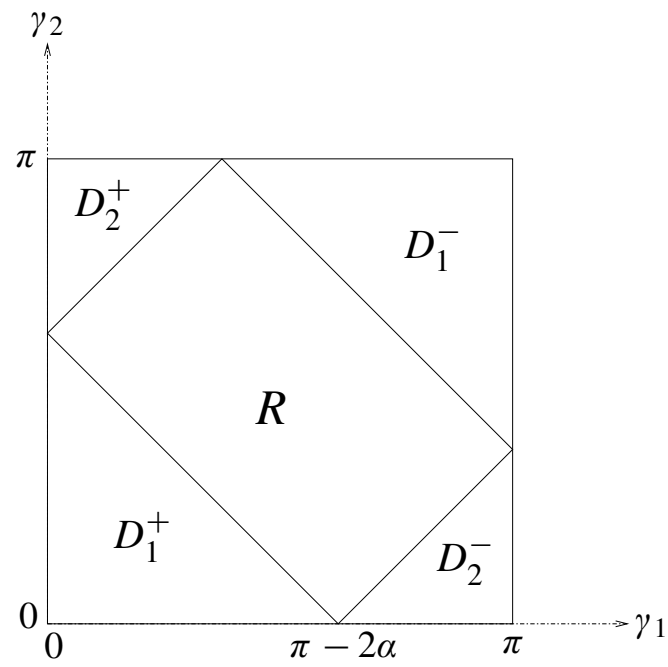

Figure 2. The Concus-Finn rectangle.

discovered bounded solutions of (3) and (2) in domains with corners whose unit normals (that is, Gauss maps) cannot extend continuously as functions of $(x, y)$ to a corner on the boundary of the domain (for example [Finn 1988b; Finn 1988a, page 15; Concus and Finn 1996, Example 2; Finn 1996]). In 1992, as a result of computational experiments, they formulated a conjecture on the continuity of such surfaces [Concus et al. 1992; Concus and Finn 1996, page 67]; additional numerical experiments in 1994 by Concus and Finn and in 1996 by Mittelmann and Zhu found evidence to support the conjecture, which says that if $\left(\gamma_{1}, \gamma_{2}\right) \in D_{2}^{ \pm}$, then $f$ has a jump discontinuity at $O$ [Finn 1999, page 776]. Writing the conditions for a pair of angles to be in $D_{2}^{ \pm}$yields the following formulation of the conjecture:

Concus-Finn conjecture. Suppose that $0<\alpha<\pi / 2$, that the limits (4) exist and that $0<\gamma_{1}, \gamma_{2}<\pi$. If $2 \alpha+\left|\gamma_{1}-\gamma_{2}\right|>\pi$, then any solution of (1) and (2), with $H(x, z)=\kappa z+\lambda$ and $\kappa$ nonnegative, has a jump discontinuity at $O$.

We will prove this conjecture when $\partial \Omega \backslash\{(0,0)\}$ is locally Hölder continuously differentiable and $\gamma$ is locally Hölder continuous on $\partial \Omega \backslash\{(0,0)\}$ in a neighborhood of the origin. For convenience, we will adopt the following notation throughout this paper. We will write points of $\mathbb{R}^{2}$ as lower case letters (for example, $x$ ) and points of $\mathbb{R}^{3}$ as upper case letters (for example, $X$ ). For $m \in \mathbb{N}$ with $m \geq 2$, we will write $O_{m}$ as the origin in $\mathbb{R}^{m}$; however, we will write $O$ for $O_{2}=(0,0)$. We denote by $B^{m}(P, r)$ the open ball in $\mathbb{R}^{m}$ of radius $r>0$ centered at $P \in \mathbb{R}^{m}$ and by $B(x, r)$ the ball $B^{2}(x, r)$ for $x \in \mathbb{R}^{2}$. We will fix $\rho^{*} \in(0,1)$ and $\alpha \in(0, \pi]$; later we will assume $\alpha \leq \pi / 2$. We will write $\omega(\theta)$ for $(\cos (\theta), \sin (\theta))$ for $\theta \in \mathbb{R}$. 
Our domain $\Omega$ will be a connected, simply connected open set in $\mathbb{R}^{2}$ such that $O \in \partial \boldsymbol{\Omega}, \partial \boldsymbol{\Omega} \backslash\{O\}$ is a piecewise $C^{1}$ curve, $\partial \boldsymbol{\Omega}$ has a corner of size $2 \alpha$ at $O$, and the tangent cone to $\partial \Omega$ at $O$ is $L^{+} \cup L^{-}$, where polar coordinates relative to $O$ are denoted by $r$ and $\theta$, and $L^{+}=\{\theta=\alpha\}$ and $L^{-}=\{\theta=-\alpha\}$. We will assume there exists $\delta^{*}>0$ such that $\partial^{+} \Omega=\partial \Omega \cap \overline{B\left(O, 3 \delta^{*}\right)} \cap T^{+}$and $\partial^{-} \Omega=\partial \Omega \cap \overline{B\left(O, 3 \delta^{*}\right)} \cap T^{-}$ are connected, $C^{1, \rho^{*}}$ arcs such that the tangent rays to $\partial^{+} \Omega$ and $\partial^{-} \Omega$ at $O$ are $L^{+}$ and $L^{-}$respectively; here $T^{+}=\left\{x \in \mathbb{R}^{2}: x_{2} \geq 0\right\}$ and $T^{-}=\left\{x \in \mathbb{R}^{2}: x_{2} \leq 0\right\}$. We set $\Lambda=\partial \boldsymbol{\Omega} \backslash\left(\partial^{+} \boldsymbol{\Omega} \cup \partial^{-} \boldsymbol{\Omega}\right)$ and obtain

$$
\partial \boldsymbol{\Omega}=\partial^{+} \boldsymbol{\Omega} \cup \partial^{-} \boldsymbol{\Omega} \cup \Lambda \quad \text { with } O \in \partial^{+} \boldsymbol{\Omega} \cap \partial^{-} \boldsymbol{\Omega} \text { and } B\left(O, 3 \delta^{*}\right) \cap \bar{\Lambda}=\varnothing .
$$

We will assume $\Omega \subset\{r \omega(\theta): r>0,-\pi<\theta<\pi\}$. Let us define $\tau^{+} \in C^{0, \rho^{*}}\left(\partial^{+} \Omega\right)$ and $\tau^{-} \in C^{0, \rho^{*}}\left(\partial^{-} \Omega\right)$ such that $\tau^{+}(O)=\alpha, \tau^{-}(O)=-\alpha$,

$$
\left(\cos \left(\tau^{+}(x)\right), \sin \left(\tau^{+}(x)\right), 0\right) \quad \text { is a unit tangent to } \partial^{+} \Omega \times \mathbb{R} \text { for } x \in \partial^{+} \Omega
$$

and

$\left(\cos \left(\tau^{-}(x)\right), \sin \left(\tau^{-}(x)\right), 0\right) \quad$ is a unit tangent to $\partial^{-} \Omega \times \mathbb{R}$ for $x \in \partial^{-} \Omega$.

We will assume (4) holds and that $\gamma \in C^{0, \rho^{*}}\left(\overline{\partial^{+} \Omega}\right)$ (when $\gamma(O)$ is set equal to $\gamma_{1}$ ) and $\gamma \in C^{0, \rho^{*}}\left(\overline{\partial^{-} \Omega}\right)$ (when $\gamma(O)$ is set equal to $\gamma_{2}$.) If $\gamma_{1}=\pi / 2$ or $\gamma_{2}=\pi / 2$, we will need to be able to use slicing [Allard 1972, 4.10] and so we will assume

$$
|D \gamma| \in L^{1}\left(\partial^{+} \Omega\right) \quad \text { if } \gamma_{1}=\frac{1}{2} \pi \quad \text { and } \quad|D \gamma| \in L^{1}\left(\partial^{-} \Omega\right) \quad \text { if } \gamma_{2}=\frac{1}{2} \pi .
$$

We will also assume $(\gamma, \Omega, O)$ is admissible as defined in Definition 3.4 (which essentially says Emmer's (boundary) condition holds at each point of $\partial \Omega \backslash\{O\}$ ). For a solution $f \in C^{2}(\Omega) \cap C^{1, \rho^{*}}(\bar{\Omega} \backslash\{O\})$ of (1) and (2), we let

$$
\vec{n}(X)=\vec{n}_{f}(X)=\frac{(\nabla f(x),-1)}{\sqrt{1+|\nabla f(x)|^{2}}}, \quad \text { where } X=(x, t) \in(\bar{\Omega} \backslash\{O\}) \times \mathbb{R},
$$

denote the downward unit normal to the graph of $f$; in the capillary interpretation, $\vec{n}$ represents the inward unit normal with respect to the fluid region. Using comparison theorems (for example, [Finn 1986, Theorem 5.1]) and existence and regularity theorems for variational solutions (for example, [Finn 1986, Theorem 7.5 together with Lemma 4.1]), we see that we may assume $f$ is a variational $(B V(\Omega))$ solution. Since our interest will be in the local behavior of solutions of (1) and (2) near the corner $O$, we sometimes think of $\Omega$ as the intersection of a larger domain with an appropriate neighborhood of $O$ and a solution $f$ of (1) and (2) as the restriction to $\bar{\Omega} \backslash\{O\}$ of a function $F$ that is a solution of a boundary value problem, perhaps like (1) and (2), in this larger domain; in this case, restricting the problem to a subdomain $\Omega$ for which $(\gamma, \Omega, O)$ is admissible is straightforward.

The following theorem will establish the validity of the Concus-Finn conjecture. 
Theorem 1.1. Let $\Omega$ and $\gamma$ be as above with $\alpha \in(0, \pi / 2]$, and suppose that $f \in C^{2}(\Omega) \cap C^{1, \rho^{*}}(\bar{\Omega} \backslash\{O\})$ is a bounded solution to (1) satisfying (2) on $\partial^{ \pm} \Omega \backslash\{O\}$ with $|H|_{\infty}=\sup _{x \in \Omega}|H(\boldsymbol{x}, f(\boldsymbol{x}))|<\infty$. Suppose (4) holds and $\gamma_{1}, \gamma_{2} \in(0, \pi)$. Then $f$ is discontinuous at $O$ whenever $\left(\gamma_{1}, \gamma_{2}\right)$ satisfies

$$
2 \alpha+\left|\gamma_{1}-\gamma_{2}\right|>\pi
$$

Notice that we exclude cases in which $\gamma_{1}$ or $\gamma_{2}$ equals 0 or $\pi$. It seems likely that an argument in this exceptional situation might use ideas from [Finn 1988b], and it would be interesting to see the details of a proof.

For linear elliptic partial differential equations, especially uniformly elliptic equations, the qualitative behavior "at" a boundary point of the solution $f$ of a boundary value problem can be determined by local information such as the prescribed boundary information and bounds on the maximum rate at which $|f|$ can go to infinity "at" the boundary point (for example, [Bear and Hile 1983]). However this is usually not true for quasilinear equations. The Concus-Finn conjecture, if true, represents one of the rare situations when the qualitative behavior of a solution (that is, its continuity at a convex corner) is determined by the boundary information (that is, $\alpha, \gamma_{1}$ and $\gamma_{2}$ ) in an arbitrarily small neighborhood of the boundary point. At a nonconvex corner $O$ (that is, $\alpha>\pi / 2$ ), [Shi and Finn 2004] shows that information about $\partial \Omega \cap B_{\epsilon}(O)$ and $\gamma$ in $B_{\epsilon}(O)$ for some $\epsilon>0$ need not be sufficient to determine the continuity at $O$ of a solution of (3) and (2).

Lancaster and Siegel [1996a] investigated the behavior of bounded solutions of (3) and (2) at corners, both convex and nonconvex corners, and they noted in [1996a; 1996b] that the conclusions in [1996a] carry over to solutions of (1) and (2) when $H$ satisfies some minor restrictions (that is, $H(\boldsymbol{x}, z)$ is either real-analytic or strictly increasing in $z)$; in this case, a bounded solution $f \in C^{2}(\Omega) \cap C^{1}(\bar{\Omega} \backslash\{O\})$ of (1) satisfying (2) on $\partial^{ \pm} \Omega \backslash\{O\}$ is in $C^{0}(\bar{\Omega})$ when $\left(\gamma_{1}, \gamma_{2}\right) \in \bar{R} \cap(0, \pi) \times(0, \pi)$. The arguments in [Concus and Finn 1996] and [Finn 1996] continue to show that if $\left(\gamma_{1}, \gamma_{2}\right) \in D_{1}^{ \pm}$, then either (1) and (2) has no solution in a neighborhood of $O$ or $f$ is unbounded in any neighborhood of $O$ when $H$ satisfies some extremely minor restrictions. Thus, under mild restrictions on $H$, Figure 2 continues to illustrate the behavior at $O$ of solutions of (1) and (2). (See Remark 3.1 for a comment about [Lancaster and Siegel 1996a].)

Once we know that a solution of (1) and (2) is discontinuous at a convex corner $O=(0,0)$, it is natural to ask about its behavior nearby. In [Lancaster and Siegel 1996a, Theorem 1], it is proven that if $\epsilon \leq \gamma \leq \pi-\epsilon$ for some $\epsilon>0$, then the radial limits of $f$,

$$
R f(\theta)=\lim _{r \downarrow 0} f(r \cos (\theta), r \sin (\theta)),
$$


exist for all $\theta \in(-\alpha, \alpha)$ and $R f \in C^{0}([-\alpha, \alpha])$, where $R f(-\alpha)$ and $R f(\alpha)$ are the limits of the trace of $f$ on $\partial^{-} \Omega$ and $\partial^{+} \Omega$ respectively; the continuity of the trace of $f$ on $\overline{\partial^{-} \Omega}$ and on $\overline{\partial^{+} \Omega}$ is a conclusion of this theorem.

Now suppose (4) holds and $2 \alpha+\left|\gamma_{1}-\gamma_{2}\right|>\pi$. Then Theorem 1.1 above and [Lancaster and Siegel 1996a, Theorems 1 and 2] imply that there exist $\alpha_{1}$ and $\alpha_{2}$ with $-\alpha<\alpha_{1}<\alpha_{2}<\alpha$ such that

$$
R f(\theta)= \begin{cases}\text { constant } & \text { if }-\alpha \leq \theta \leq \alpha_{1}, \\ \text { strictly monotonic } & \text { if } \alpha_{1} \leq \theta \leq \alpha_{2} \\ \text { constant } & \text { if } \alpha_{2} \leq \theta \leq \alpha\end{cases}
$$

and $\alpha_{1}-(-\alpha) \geq \pi-\gamma_{2}$ and $\alpha-\alpha_{2} \geq \gamma_{1}$ if $R f$ is increasing on $\left(\alpha_{1}, \alpha_{2}\right)$, while $\alpha_{1}-(-\alpha) \geq \gamma_{2}$ and $\alpha-\alpha_{2} \geq \pi-\gamma_{1}$ if $R f$ is decreasing on $\left(\alpha_{1}, \alpha_{2}\right)$. Lancaster and Siegel [1996a] call the intervals $\left[-\alpha, \alpha_{1}\right]$ and $\left[\alpha_{2}, \alpha\right]$ fans (of constant radial limits), due to the shape of a region $\left\{(r \cos (\theta), r \sin (\theta)): r>0, \alpha_{2} \leq \theta \leq \alpha\right\}$ on whose closure $f$ is continuous; for nonconvex corners, a central fan (of constant radial limits) with size $\pi$ can also exist. In particular, we see that Theorem 1.1 implies $f$ has a jump discontinuity at $O$.

This work arose as a consequence of the Summer School on Capillarity held at the Max-Planck-Institut für Mathematik in Leipzig in June and July of 2003. While the Concus-Finn conjecture was discussed at meetings prior to 2003 (for example, the International Conference on Differential Equations and Dynamic Systems, University of Waterloo, Waterloo, Canada, August, 1997), the 2003 summer school brought together experts such as Maria Athanassenas, Robert Finn, Kirk Lancaster, John McCuan, Erich Miersemann, David Siegel, Tom Vogel and Henry Wente. In particular, Athanassenas and I worked (unsuccessfully) to find a counterexample to the Concus-Finn conjecture while others attempted to find a proof; our failure to find a counterexample together with the strong confidence in the correctness of the conjecture by others, especially John McCuan, inspired me to attempt to prove the conjecture. After the idea for a proof in the zero mean curvature case was obtained in 2004, Robert Finn strongly encouraged me to find a proof in the general case. In 2005, I did discover the idea of a proof; modulo some essentially minor technical modifications, this idea forms the basis for this work. This discovery may not have happened without the contributions of Athanassenas, Finn and McCuan. On the other hand, the absence of a subsequent summer school on capillarity, perhaps in the United States, may have delayed progress on important questions in capillarity (for example, [Athanassenas and Lancaster 2008; Finn 1999, 2002b; 2002a].)

\section{Image of the Gauss map}

In this section, we characterize in Theorem 2.1 the behavior of the limits at points of $\{O\} \times \mathbb{R}$ of the Gauss map for the graph of $f$. The proof involves the use 
of a 1975 result by Massari and Pepe [1975], generalized solutions (for example, [Giusti 1980]) and Leon Simon's capillarity paper [1980].

The following proposition is [Massari and Pepe 1975, Theorem 3], provided in translation for the convenience of the reader; the author thanks Professor Giuseppe Tenti of the Department of Applied Mathematics of the University of Waterloo for a translation of that paper. Here $\partial^{*} A$ denotes the reduced boundary of a Caccioppoli set $A$,

$$
v_{A}(x)=\lim _{\rho \rightarrow 0} \frac{\int_{B(x, \rho)} D \phi_{A}}{\int_{B(x, \rho)}\left|D \phi_{A}\right|}
$$

and $\left|v_{A}(x)\right|=1$ for $x \in \partial^{*} A$; if $\partial A$ is a $C^{1}$ hypersurface, $x \in \partial A$, and $v(x)$ is the interior unit normal to $\partial A$, then $v_{A}(x)=v(x)$; see for example [Giusti 1984, Chapter 3]. In the proposition, $v_{h}(x)$ denotes $v_{E_{h}}(x), v(x)$ denotes $v_{E}(x)$, and $\Omega$ denotes an open set in $\mathbb{R}^{n}$; in the context used in this paper, such an open set might be $B^{3}(X, r)$ for $X \in \mathbb{R}^{3}$ and $r>0$, or $\Omega_{\infty} \times \mathbb{R}$.

Proposition 2.1. Let $\left\{E_{h}\right\}_{h}$ be a sequence of Caccioppoli sets of mean curvature $A_{h} \in L_{\mathrm{loc}}^{p}(\Omega)$ with $p>n$. If

$$
\begin{aligned}
\phi_{E_{h}}(x) & \rightarrow \phi_{E}(x) \quad \text { in } L_{\mathrm{loc}}^{1}(\Omega), \\
\partial E_{h} \cap \Omega \ni x_{h} & \rightarrow x \in \partial^{*} E \cap \Omega, \\
A_{h}(x) & \rightarrow A(x) \quad \text { in } L_{\mathrm{loc}}^{1}(\Omega)
\end{aligned}
$$

and if for every compact $K$ of $\Omega$ there exists a constant $\gamma(K)$ such that

$$
\left\|A_{h}\right\|_{L^{p}(K)}<\gamma(K) \quad \text { for all } h \in \mathbb{N},
$$

then there exists $h_{0} \in \mathbb{N}$, such that, for every $h>h_{0}$, we have

$$
\begin{aligned}
x_{h} & \in \partial^{*} E_{h} \cap \Omega, \\
\lim _{h \rightarrow \infty} v_{h}\left(x_{h}\right) & =v(x) .
\end{aligned}
$$

Remark 2.1. We define densities in the usual manner. If $\mu$ is a measure on $\mathbb{R}^{n}$ and $a \in \mathbb{R}^{n}$, we define the $m$-dimensional upper density $\Theta^{* m}(\mu, a)$, lower density $\Theta_{*}^{m}(\mu, a)$ and density $\Theta^{m}(\mu, a)$ of $\mu$ at $a$ as in [Allard 1972]. For example,

$$
\Theta^{* m}(\mu, x)=\limsup _{r \downarrow 0} \frac{\mu\left(B^{n}(x, r)\right)}{\alpha_{m} r^{m}} .
$$

If $A \subset \mathbb{R}^{n}, \quad x \in \mathbb{R}^{n}$ and $m \leq n$, we define the $m$-dimensional upper (mass) density $\Theta^{* m}(A, x)$, the $m$-dimensional lower (mass) density $\Theta_{*}^{m}(A, x)$ and the $m$ dimensional (mass) density $\Theta^{m}(A, x)$ of $A$ at $x$ in the usual way. For example,

$$
\Theta_{*}^{m}(A, x)=\liminf _{r \downarrow 0} \frac{H^{m}\left(B^{n}(x, r) \cap A\right)}{\alpha_{m} r^{m}} ;
$$


here $\alpha_{m}=H^{m}\left(B^{m}\left(O_{m}, 1\right)\right)$ denotes the $m$-dimensional volume of the unit ball in $\mathbb{R}^{m}$.

Recall that a $m$-dimensional varifold in $\mathbb{R}^{n}$ is a Radon measure on $\mathbb{R}^{n} \times \mathrm{G}(n, m)$. We denote the space of $m$-dimensional varifolds in $\mathbb{R}^{n}$ (with the weak topology) by $\mathbf{V}_{m}\left(\mathbb{R}^{n}\right)$. To each $\mathscr{H}^{m}$ measurable and $\left(\mathscr{H}^{m}, m\right)$ rectifiable set $S$ in $\mathbb{R}^{n}$ is associated a varifold (for example [Allard 1972, Sections 3.5 and 4.7; Taylor 1976, Section I]); we adopt the notation $\mathbf{v}(S)$ of [Allard 1972] for this varifold, whereas [Taylor 1976] uses the notation $|S|$. We denote the first variation of $V \in \mathbf{V}_{m}\left(\mathbb{R}^{n}\right)$ by $\delta V$, as in [Allard 1972, Chapter 4].

For $r>0$, let $\mu_{r}: \mathbb{R}^{n} \rightarrow \mathbb{R}^{n}$ be defined by $\mu_{r}(X)=r X$ for $X \in \mathbb{R}^{n}$. Let $V \in \mathbf{V}_{m}\left(\mathbb{R}^{n}\right)$. We set $V_{r}=\mu_{r \#} V$ (for example [Allard 1972, Section 3.2; Taylor 1976, Section I]); then

$$
\left\|V_{r}\right\|=r^{m} \mu_{r \#}\|V\| \quad \text { and } \quad\left\|\delta V_{r}\right\|=r^{m-1} \mu_{r \#}\|\delta V\|
$$

by [Allard 1972, 3.2(2) and 4.12(1)], respectively. Notice that if $L>0$, then

$$
\begin{aligned}
\left\|\mu_{r \#} V\right\|\left(B\left(O_{n}, L\right)\right) & =r^{m} \mu_{r \#}\|V\|\left(B\left(O_{n}, L\right)\right) \\
& =r^{m}\|V\|\left(B\left(O_{n}, L / r\right)\right)=L^{m} \frac{\|V\|\left(B\left(O_{n}, L / r\right)\right)}{(L / r)^{m}} .
\end{aligned}
$$

Thus, if $\Theta^{* m}\left(\|V\|, O_{n}\right)<\infty$,

$$
\limsup _{r \rightarrow \infty}\left\|\mu_{r \#} V\right\|\left(B\left(O_{n}, L\right)\right) \leq L^{m} \alpha(m) \Theta^{* m}\left(\|V\|, O_{n}\right) .
$$

Similarly, if $k=m-1$ and $\Theta^{* k}\left(\|\delta V\|, O_{n}\right)<\infty$, then

$$
\limsup _{r \rightarrow \infty}\left\|\delta\left(\mu_{r \#} V\right)\right\|\left(B \left(\left(O_{n}, L\right) \leq L^{k} \alpha(k) \Theta^{* k}\left(\|\delta V\|, O_{n}\right) .\right.\right.
$$

Theorem 2.1. Suppose $\Omega$ and $\gamma$ are as in Theorem 1.1 such that (4) holds with $\gamma_{1}, \gamma_{2} \in(0, \pi)$ and $\gamma_{2}-\gamma_{1}>\pi-2 \alpha$, that is, $\left(\gamma_{1}, \gamma_{2}\right) \in D_{2}^{+}$. Let $f \in C^{2}(\Omega) \cap$ $C^{1, \rho^{*}}(\bar{\Omega} \backslash\{O\})$ be a bounded solution of (1) and (2) and suppose there exists $J \in(0, \infty)$ such that $|H(x, f(x))| \leq J$ on $\Omega \times \mathbb{R}$. Let $\beta \in(-\alpha, \alpha)$ and let $\left(x_{j}\right)$ be a sequence in $\Omega$ satisfying $\lim _{j \rightarrow \infty} x_{j}=O$ and

$$
\lim _{j \rightarrow \infty} x_{j} /\left|x_{j}\right|=(\cos (\beta), \sin (\beta)) .
$$

(i) If $\beta \in\left[-\alpha+\pi-\gamma_{2}, \alpha-\gamma_{1}\right]$, then $\lim _{j \rightarrow \infty} \vec{n}\left(x_{j}\right)=(-\sin (\beta), \cos (\beta), 0)$.

(ii) If $\beta \in\left(-\alpha,-\alpha+\pi-\gamma_{2}\right]$, then

$$
\lim _{j \rightarrow \infty} \vec{n}\left(x_{j}\right)=\left(-\sin \left(-\alpha+\pi-\gamma_{2}\right), \cos \left(-\alpha+\pi-\gamma_{2}\right), 0\right) .
$$

(iii) If $\beta \in\left[\alpha-\gamma_{1}, \alpha\right)$, then $\lim _{j \rightarrow \infty} \vec{n}\left(x_{j}\right)=\left(-\sin \left(\alpha-\gamma_{1}\right), \cos \left(\alpha-\gamma_{1}\right), 0\right)$. 
The proof consists of minor modifications of the proof of [Simon 1980] and the use of generalized solutions [Giusti 1980; Jeffres and Lancaster 2007]. The rationale for using results from [Simon 1980] and [Giusti 1980] is essentially the same as that used in [Tam 1986c]. (See Remark 2.3.) Simon's technique is the standard one (for example, [Federer 1969, Sections 3.1 and 5.4]) of blowing up the graph of a solution of (1) and (2) about the origin $O_{3} \in \mathbb{R}^{3}$; Simon obtains a plane through the origin, and we modify that proof to show that the limit of a blowup about $O_{3}$ of the graph of $f-R f(\beta)$ is a vertical half-plane $\pi_{1}$. Unfortunately, the third component of the image $\left(x_{j_{k}} / \epsilon_{j_{k}},\left[f\left(\epsilon_{j_{k}} x_{j_{k}}\right)-R f(\beta)\right] / \epsilon_{j_{k}}\right)$ of the blowup sequence being used might diverge to infinity. We therefore consider a type of sequence introduced in [Tam 1986c] and use the result above, Proposition 2.1 and $B V(\Omega \times \mathbb{R})$ techniques (for example, [Jeffres and Lancaster 2007]) to determine the unit normal to $\pi_{1}$. One might wish to read Remark 2.4 before examining the proof of this theorem.

It will be convenient to define some quantities and state an assumption. Set

$$
\begin{array}{llrl}
\epsilon_{0} & =\frac{1}{8} \min \left\{\gamma_{1}, \pi-\gamma_{1}, \gamma_{2}, \pi-\gamma_{2}\right\}, & \zeta=\frac{1}{2} \pi-2 \epsilon_{0}, \\
c_{1}=\frac{1}{4}\left(\cos \left(2 \epsilon_{0}\right)-\left|\cos \left(\gamma_{1}\right)\right|\right), & \lambda_{1}=(\cos (\alpha-\zeta), \sin (\alpha-\zeta), 0), \\
c_{2}=\frac{1}{4}\left(\cos \left(2 \epsilon_{0}\right)-\left|\cos \left(\gamma_{1}\right)\right|\right), & \lambda_{2}=(\cos (-\alpha+\zeta), \sin (-\alpha+\zeta), 0), \\
C=\left(\min \left\{\sin \left(\epsilon_{0}\right), c_{1}, c_{2}\right\}\right)^{-1} . &
\end{array}
$$

A quick calculation shows $\lim \inf _{\partial+\Omega \ni x \rightarrow 0}\left(-v(x) \cdot \lambda_{1}+\cos (\gamma(x)) \vec{n}(x) \cdot \lambda_{1}\right) \geq 4 c_{1}$ and $\liminf _{\partial^{-} \Omega \ni x \rightarrow 0}\left(-v(x) \cdot \lambda_{2}+\cos (\gamma(x)) \vec{n}(x) \cdot \lambda_{2}\right) \geq 4 c_{2}$. We will assume $\delta^{*}>0$ was chosen small enough that

(a) $\left|\tau^{+}(x)-\alpha\right|<\alpha / 4$ and $\left|\tau^{-}(x)+\alpha\right|<\alpha / 4$ if $|x| \leq 3 \delta^{*}$.

(b) $\Omega \cap B\left(O, 3 \delta^{*}\right) \subset\left\{r \omega(\theta): r>0, \theta \in\left[-\alpha-\epsilon_{0}, \alpha+\epsilon_{0}\right]\right\}$.

(c) $-v(x) \cdot \lambda_{1}+\cos (\gamma(x)) \vec{n}(x) \cdot \lambda_{1} \geq 2 c_{1}$ if $x \in \partial^{+} \Omega$ and $|x| \leq 3 \delta^{*}$.

(d) $-v(x) \cdot \lambda_{2}+\cos (\gamma(x)) \vec{n}(x) \cdot \lambda_{2} \geq 2 c_{2}$ if $x \in \partial^{-} \Omega$ and $|x| \leq 3 \delta^{*}$.

Notice that (a) and (b) imply there exist $x^{ \pm}:\left[0,3 \delta^{*}\right] \rightarrow \mathbb{R}^{2}$ that are parametrizations of $\partial^{ \pm} \Omega$ such that $x=x^{+}(|x|)$ for $x \in \partial^{+} \Omega$ and $x=x^{-}(|x|)$ for $x \in \partial^{-} \Omega$. Let $\Omega_{\lambda}=\Omega \cap B(O, \lambda)$ for $\lambda>0$.

Proof. Consider $\beta \in(-\alpha, \alpha)$ fixed and set $u(x)=f(x)-R f(\beta)$, as in [Lancaster and Siegel 1996a]. Set $\delta_{0}=2 \delta^{*}$. Let

$$
U=\{(x, t): x \in \Omega, t<u(x)\} \quad \text { be the subgraph of } u
$$


and

$$
\begin{aligned}
\mathcal{M}_{0} & =\left\{(x, u(x)): x \in \Omega \cap B\left(O, 3 \delta^{*}\right)\right\}, \\
\mathcal{M} & =\left\{(x, u(x)): x \in \overline{\Omega \cap B\left(O, 3 \delta^{*}\right)} \backslash\{O\}\right\}, \\
\partial^{+} \mathcal{M} & =\left\{(x, u(x)): x \in \partial^{+} \Omega \backslash\{O\}\right\}, \\
\partial^{-} M & =\left\{(x, u(x)): x \in \partial^{-} \Omega \backslash\{O\}\right\} .
\end{aligned}
$$

Notice that $\partial U \cap\left(\Omega_{3 \delta^{*}} \times \mathbb{R}\right)=M_{0}$. Let $V=\mathbf{v}(M)$ and $V_{0}=\mathbf{v}\left(M_{0}\right)$ and note that these are both two-dimensional integral varifolds; see for example [Allard 1972, Section 3.5].

We will first use a variation of the argument in [Simon 1980, Section 1] $]^{1}$ to show that

$$
\mathscr{H}^{1}\left(\partial^{+} \mu \cup \partial^{-} \mathcal{M}\right)<\infty .
$$

As in [S], let $\eta$ denote the unit vector normal to $\partial M=\partial^{+} \mu \cup \partial^{-} M$ that is tangent to $M$ and points into $\Omega \times \mathbb{R}$; in the notation here,

$$
\eta(X)=\frac{-v(X)+(\vec{n}(X) \cdot v(X)) \vec{n}(X)}{|-v(X)+(\vec{n}(X) \cdot v(X)) \vec{n}(X)|}=\frac{-v(X)+\cos (\gamma) \vec{n}(X)}{|-v(X)+\cos (\gamma) \vec{n}(X)|} .
$$

Let $h_{1}, h_{2}, s \in C^{\infty}(\mathbb{R})$ with $0 \leq h_{1}(t), h_{2}(t), s(t) \leq 1$ for $t \in \mathbb{R}$, such that $h_{1}=0$ on $(-\infty,-\alpha / 2]$ and $h_{1}=1$ on $[\alpha / 2, \infty)$, with $h_{2}=1-h_{1}$ and $s(t)=1$ if $|t| \leq 2 \delta^{*}$ and $s(t)=0$ if $|t| \geq 3 \delta^{*}$. Define $\phi_{1}, \phi_{2} \in C^{\infty}((\bar{\Omega} \backslash\{O\}) \times \mathbb{R})$ such that

$$
\phi_{1}(r \omega(\theta), z)=h_{1}(\theta) s(r) \lambda_{1} \quad \text { and } \quad \phi_{2}(r \omega(\theta), z)=h_{2}(\theta) s(r) \lambda_{2}
$$

for $0<r<\infty$ and $\theta \in(-\pi, \pi)$ that satisfy $r \omega(\theta) \in \bar{\Omega} \backslash\{O\}$. Notice that $\sup r\left|D \phi_{1}\right|<\infty$ and $\sup r\left|D \phi_{2}\right|<\infty$. As in [S, (1.4)], we obtain

$$
\begin{aligned}
\rho^{-1} \int_{\mathcal{M}[B(O, \rho) \times \mathbb{R}]}\left(\phi_{1} \cdot \delta^{M} r\right) d \mathscr{H}^{2}+ & \int_{\partial^{+} \mu} \min \{r / \rho, 1\} \phi_{1} \cdot \eta d \mathscr{H}^{1} \\
& =-\int_{\mu} \min \{r / \rho, 1\}\left(\delta^{M} \cdot \phi_{1}+H v \cdot \phi_{1}\right) d \mathscr{H}^{2},
\end{aligned}
$$

since $h_{1}(t)=0$ if $t \leq-\alpha / 2$, and

$$
\begin{aligned}
\rho^{-1} \int_{M \cap B(O, \rho) \times \mathbb{R}]}\left(\phi_{2} \cdot \delta^{M} r\right) d \mathscr{H}^{2}+ & \int_{\partial^{-} \mu} \min \{r / \rho, 1\} \phi_{2} \cdot \eta d \mathscr{H}^{1} \\
& =-\int_{\mu} \min \{r / \rho, 1\}\left(\delta^{M} \cdot \phi_{2}+H v \cdot \phi_{2}\right) d \mathscr{H}^{2},
\end{aligned}
$$

since $h_{2}(t)=0$ if $t \geq \alpha / 2$. From (b) and (c), we see that

$$
\phi_{1}(X) \cdot \eta(X) \geq c_{1} h_{1}(\theta) s(r) \quad \text { if } X=(r \omega(\theta), z) \in \partial^{+} \mu \text { with } \theta \in\left(0, \alpha+\epsilon_{0}\right)
$$

\footnotetext{
${ }^{1}$ In this proof, we refer to [Simon 1980] as [S].
} 
and, from (b) and (d), that $\phi_{2}(X) \cdot \eta(X) \geq c_{2} h_{2}(\theta) s(r) \quad$ if $X=(r \omega(\theta), z) \in \partial^{-} \mathcal{M}$ with $\theta \in\left(-\alpha-\epsilon_{0}, 0\right)$.

Using the argument on [S, page 367], we obtain

$$
\mathscr{H}^{1}\left(\partial^{+} \mu \cap\left(B\left(O, \delta_{0}\right) \times \mathbb{R}\right)\right)<\infty \text { and } \mathscr{H}^{1}\left(\partial^{-} \mu \cap\left(B\left(O, \delta_{0}\right) \times \mathbb{R}\right)\right)<\infty \text {. }
$$

Since $f$, and so $u$, is in $C^{1, \rho^{*}}(\bar{\Omega} \backslash\{O\})$, we see that (17) holds.

As in the proof of [S, (1.8)], we see using [Allard 1972, 4.2, 4.3(5), 4.7] that (1), (17) and [S, (1.1)] imply

$$
\|\delta V\|(B(O, r) \times \mathbb{R}) \leq J \mathscr{H}^{2}(\mathcal{M} \cap(B(O, r) \times \mathbb{R}))+\mathscr{H}^{1}(\partial \mathcal{M} \cap(B(O, r) \times \mathbb{R}))
$$

and therefore

$$
\|\delta V\|(B(O, r) \times \mathbb{R})<\infty \quad \text { for } 0<r<R_{2} .
$$

Set $K=\max \left\{\sup _{\Omega \times \mathbb{R}}\left|\delta^{M} \cdot \phi_{1}\right|, \sup _{\Omega \times \mathbb{R}}\left|\delta^{M} \cdot \phi_{2}\right|\right\}$.

Now let us substitute in [S, (1.4)] successively $\phi=\phi_{1} \psi$ and $\phi=\phi_{2} \psi$, where $\psi \in C_{0}^{1}\left(B\left(O, 3 \delta^{*}\right) \times \mathbb{R}\right)$. If we argue as in [S], we obtain the following analogues for $k=1,2$ of [S, (1.10)]:

$$
\begin{aligned}
& \rho^{-1} \int_{M \cap(B(O, \rho) \times \mathbb{R})} \psi\left(\phi_{k} \cdot D r\right) d \mathscr{H}^{2}+\int_{\partial \mathcal{M}} \psi\left(\phi_{k} \cdot \eta\right) d \mathcal{H}^{1} \\
&\leq(K+J)) \int_{\mathcal{M}}\left(\psi+\left|\delta^{M} \psi\right|\right) d \mathcal{H}^{2}+o(1) \quad \text { as } \rho \rightarrow 0 .
\end{aligned}
$$

Now (b) implies $\lambda_{1} \cdot D r \geq \sin \left(\epsilon_{0}\right)$ on the support of $\phi_{1}$ and $\lambda_{2} \cdot D r \geq \sin \left(\epsilon_{0}\right)$ on the support of $\phi_{2}$. Therefore, if $0<\rho<\delta_{0}$, then

$$
\begin{aligned}
& \limsup _{\rho \downarrow 0} \rho^{-1} \int_{\mathcal{M}(B(O, \rho) \times \mathbb{R})} h_{1} \psi d \mathscr{H}^{2}+\int_{\partial \mathcal{M}} h_{1} \psi d \mathscr{H}^{1} \\
& \leq C(K+J) \int_{\mathcal{M}}\left(h_{1} \psi+\left|\delta^{M}\left(h_{1} \psi\right)\right|\right) d \mathscr{H}^{2}
\end{aligned}
$$

and

$$
\begin{aligned}
& \limsup _{\rho \downarrow 0} \rho^{-1} \int_{\mathcal{M}(B(O, \rho) \times \mathbb{R})} h_{2} \psi d \mathscr{H}^{2}+\int_{\partial \mathcal{M}} h_{2} \psi d \mathscr{H}^{1} \\
& \leq C(K+J) \int_{\mathcal{M}}\left(h_{2} \psi+\left|\delta^{M}\left(h_{2} \psi\right)\right|\right) d \mathscr{H}^{2} .
\end{aligned}
$$


By adding these inequalities, we see that if $0<\rho<\delta_{0}$ then

$$
\begin{aligned}
\underset{\rho \downarrow 0}{\limsup \rho^{-1}} & \int_{\mathcal{M}(B(O, \rho) \times \mathbb{R})} \psi d \mathscr{H}^{2}+\int_{\partial \mathcal{M}} \psi d \mathscr{H}^{1} \\
& \leq C(K+J) \int_{\mathcal{M}}\left(\psi+\left|\delta^{M}\left(h_{1} \psi\right)\right|+\left|\delta^{M}\left(h_{2} \psi\right)\right|\right) d \mathscr{H}^{2} \\
& \leq C(K+J) \int_{\mathcal{M}}\left(\psi\left(1+\left|\delta^{M}\left(h_{1}\right)\right|+\left|\delta^{M}\left(h_{2}\right)\right|\right)+\left|\delta^{M}(\psi)\right|\right) d \mathscr{H}^{2} .
\end{aligned}
$$

From the first part of [Allard 1972, 3.1(2)], we see this implies for the varifold $V=\mathbf{v}(M)$ that

$$
\|\delta V\|(\psi) \leq C(K+J) \int\left(\psi\left(1+\left|\delta^{M}\left(h_{1}\right)\right|+\left|\delta^{M}\left(h_{2}\right)\right|\right)+\left|\delta^{M}(\psi)\right|\right) d\|V\|,
$$

which is an analogue of [S, (1.11)]. As in [S], this implies

$$
\mathscr{H}^{2}\left(M \cap B^{3}(Y, \rho)\right) \geq \bar{C} \rho^{2}\left(1+\delta_{0}\right),
$$

for some constant $\bar{C}>0$, and therefore

$$
\Theta_{*}^{2}(\|V\|, Y) \geq \bar{C}>0
$$

if $0<\rho<\delta_{0}$ and $Y \in \bar{M} \cap(B(O, \sigma) \times \mathbb{R})$. (These two conclusions can be obtained independently using $B V(\Omega)$ techniques and Lemma 3.1.)

Let

$$
\begin{aligned}
& F_{1}=\left\{(x, t): x \in \partial^{+} \Omega \backslash\{O\}, t \leq u(x)\right\}, \\
& \tilde{F}_{1}=\left\{(x, t): x \in \partial^{+} \Omega \backslash\{O\}, t \geq u(x)\right\}, \\
& F_{2}=\left\{(x, t): x \in \partial^{-} \Omega \backslash\{O\}, t \leq u(x)\right\}, \text { and } \\
& \tilde{F}_{2}=\left\{(x, t): x \in \partial^{-} \Omega \backslash\{O\}, t \geq u(x)\right\} .
\end{aligned}
$$

Let $W_{1}=\mathbf{v}\left(F_{1}\right), \tilde{W}_{1}=\mathbf{v}\left(\tilde{F}_{1}\right), W_{2}=\mathbf{v}\left(F_{2}\right)$ and $\tilde{W}_{2}=\mathbf{v}\left(\tilde{F}_{2}\right)$, be the two-dimensional varifolds associated with $F_{1}, \tilde{F}_{1}, F_{2}$ and $\tilde{F}_{2}$, respectively (for example, [Allard 1972, Sections 3.5 and 4.7] and [Taylor 1976, Section 1]). Set

$$
E_{1}=\left\{x \in \partial \Omega: \gamma(x)<\frac{1}{2} \pi\right\} \times \mathbb{R} \quad \text { and } \quad E_{2}=\left\{x \in \partial \Omega: \gamma(x)>\frac{1}{2} \pi\right\} \times \mathbb{R} .
$$

Define $Z$ to be the two-dimensional varifold given by $Z=V-W_{1} L \cos (\gamma) \chi_{E_{2}}+\tilde{W}_{1} L \cos (\gamma) \chi_{E_{1}}-W_{2} L \cos (\gamma) \chi_{E_{2}}+\tilde{W}_{2} L \cos (\gamma) \chi_{E_{1}}$. The monotonicity formula [S, (2.6)] holds for $Z$; that is, there exists $c \geq 0$ such that

$$
\exp \left(c r^{\beta}\right) \frac{\|Z\|\left(B^{3}\left(O_{3}, r\right)\right)}{r^{2}} \quad \text { is increasing in } r \text { for } 0<r<R
$$


and, in conjunction with (21), we see that the two-dimensional density of $Z$ at $O_{3}$ exists and

$$
\Theta^{2}\left(\|Z\|, O_{3}\right) \in(0, \infty) .
$$

We note, for example, that if $\gamma_{1}=\pi / 2$, then (5) is used in a slicing argument (that is, [Allard 1972, 4.10(1)]) to show that [S, (2.3)] (with $\partial \Omega$ replaced by $\partial^{+} \Omega$ ) holds.

Suppose $\left(x_{j}\right)$ is a sequence in $\Omega$ converging to $O$ as $j \rightarrow \infty$ and satisfying (16). For each $j \in \mathbb{N}$, set $\epsilon_{j}=\left|x_{j}\right|$ and $\Omega_{j}=\left\{x \in \mathbb{R}^{2}: \epsilon_{j} x \in \Omega\right\}$, and define $f_{j}, u_{j} \in$ $C^{2}\left(\Omega_{j}\right) \cap C^{1}\left(\overline{\Omega_{j}} \backslash\{O\}\right)$ by

$$
f_{j}(x)=\frac{f\left(\epsilon_{j} x\right)-f\left(x_{j}\right)}{\epsilon_{j}} \quad \text { and } \quad u_{j}(x)=\frac{f\left(\epsilon_{j} x\right)-R f(\beta)}{\epsilon_{j}}=\frac{u\left(\epsilon_{j} x\right)}{\epsilon_{j}} ;
$$

notice that $\nabla u_{j}=\nabla f_{j}$ on $\Omega_{j}$ and $u_{j}(x)=f_{j}(x)+c_{j}$ if $c_{j}=\left(f\left(x_{j}\right)-R f(\beta)\right) / \epsilon_{j}$. Let $\vec{n}_{j}$ be the downward unit normal to the graph of $f_{j}$ (and the graph of $u_{j}$ ), so that

$$
\vec{n}_{j}(x)=\vec{n}\left(\epsilon_{j} x\right)=\left(T f_{j}(x), \frac{-1}{\sqrt{1+\left|\nabla f_{j}(x)\right|^{2}}}\right) \text { for } x \in \Omega_{j} .
$$

Let $U_{j}=\left\{(x, t) \in \Omega_{j} \times \mathbb{R}: t<u_{j}(x)\right\}$ be the subgraph of $u_{j}$ for each $j \in \mathbb{N}$. Notice that $\mu_{1 / \epsilon_{j}}\left(M_{0}\right)=\partial U_{j} \cap\left(\Omega_{j} \times \mathbb{R}\right)$ and $\mu_{1 / \epsilon_{j}}(M) \subset \partial U_{j} \cap\left(\left(\overline{\Omega_{j}} \backslash\{O\}\right) \times \mathbb{R}\right)$.

From [Allard 1972, 2.6(2)(a)] with $\mathscr{G}=\left\{B\left(O_{3}, L\right) \times G(3,2): L>0\right\}$, we see that (14) implies that there is a subsequence $\left(\epsilon_{j_{k}}\right)$ of $\left(\epsilon_{j}\right)$ and a varifold $C \in \mathbf{V}_{2}\left(\mathbb{R}^{3}\right)$ in the varifold tangent of $Z$ at $O$ such that

$$
C=\lim _{k \rightarrow \infty} Z_{1 / \epsilon_{j_{k}}},
$$

where $Z_{1 / \epsilon_{j_{k}}}=\mu_{1 / \epsilon_{j_{k}} \#}(Z)$. By (14) and [Allard 1972, 2.6(2)(c)],

$$
\|C\|\left(B\left(O_{3}, L\right)\right)=C\left(B\left(O_{3}, L\right) \times G(3,2)\right) \leq L^{2} \alpha(2) \Theta^{2}\left(\|Z\|, O_{3}\right) ;
$$

from (22), we see that $\mu_{r \#}\|C\|=\|C\|$ for all $r>0$ (as observed in [Simon 1980], p. 576). Since $\|V\|(\Omega \times \mathbb{R})=\|Z\|(\Omega \times \mathbb{R})$ and (17) holds (hence $\mathscr{H}^{2}(\mu \cap(\partial \Omega \times \mathbb{R}))=$ $0)$, we see that

$$
\Theta^{* 2}\left(\|V\|, O_{3}\right) \leq \Theta^{2}\left(\|Z\|, O_{3}\right)<\infty .
$$

Using (14) and [Allard 1972, 2.6(2)(a)], we notice that there is a subsequence of $\left(\epsilon_{j_{k}}\right)$, still denoted $\left(\epsilon_{j_{k}}\right)$, and a varifold $V_{\infty} \in \mathbf{V}_{2}\left(\mathbb{R}^{3}\right)$ in the varifold tangent of $V$ at $\mathrm{O}_{3}$ such that

$$
V_{\infty}=\lim _{k \rightarrow \infty} V_{1 / \epsilon_{j_{k}}}
$$

and, by (14) and [Allard 1972, 2.6(2)(c)],

$$
\left\|V_{\infty}\right\|\left(B\left(O_{3}, L\right)\right)=V_{\infty}\left(B\left(O_{3}, L\right) \times G(3,2)\right) \leq L^{2} \alpha(2) \Theta^{2}\left(\|Z\|, O_{3}\right) .
$$


In a similar manner (as in [S, page 370]), we see that

$$
\begin{aligned}
& W_{1, \infty}=\lim _{k \rightarrow \infty} \mu_{1 / \epsilon_{j_{k}} \#}\left(W_{1} L a\left(-\cos \left(\gamma_{1}\right)\right) \cos \left(\gamma_{1}\right) \chi_{F_{1}}\right), \\
& \tilde{W}_{1, \infty}=\lim _{k \rightarrow \infty} \mu_{1 / \epsilon_{j_{k}} \#}\left(\tilde{W}_{1} L a\left(\cos \left(\gamma_{1}\right)\right) \cos \left(\gamma_{1}\right) \chi_{\tilde{F}_{1}}\right), \\
& W_{2, \infty}=\lim _{k \rightarrow \infty} \mu_{1 / \epsilon_{j_{k}} \#}\left(W_{2} L a\left(-\cos \left(\gamma_{2}\right)\right) \cos \left(\gamma_{2}\right) \chi_{F_{2}}\right), \\
& \tilde{W}_{2, \infty}=\lim _{k \rightarrow \infty} \mu_{1 / \epsilon_{j_{k}} \#}\left(\tilde{W}_{2} L a\left(\cos \left(\gamma_{2}\right)\right) \cos \left(\gamma_{2}\right) \chi_{\tilde{F}_{2}}\right)
\end{aligned}
$$

all exist and

$$
C=V_{\infty}-\cos \left(\gamma_{1}\right) W_{1, \infty}+\cos \left(\gamma_{1}\right) \tilde{W}_{1, \infty}-\cos \left(\gamma_{2}\right) W_{2, \infty}+\cos \left(\gamma_{2}\right) \tilde{W}_{2, \infty} .
$$

Notice that $W_{1, \infty}=0$ if $\cos \left(\gamma_{1}\right)<0$ and $\tilde{W}_{1, \infty}=0$ if $\cos \left(\gamma_{1}\right)>0$, and that $W_{2, \infty}=0$ if $\cos \left(\gamma_{2}\right)<0$ and $\tilde{W}_{2, \infty}=0$ if $\cos \left(\gamma_{2}\right)>0$.

Using the arguments in [S, Section 3 up to the top of page 373 (including (3.5)')], we see the following.

(i) For each $\rho>0$, there is a sequence $\left\{\delta_{k}\right\}$ of positive reals that converges to zero such that

$$
B^{3}\left(O_{3}, \rho\right) \cap M_{j_{k}} \subset\left\{Y \in B^{3}\left(O_{3}, \rho\right): \operatorname{dist}\left(Y, \operatorname{spt}\left(\left\|V_{\infty}\right\|\right)\right)<\delta_{k}\right\},
$$

where $M_{j_{k}}=\mu_{1 / \epsilon_{j_{k}}} M$ for each $k \in \mathbb{N}$ (that is, [S, (2.7)].)

(ii) $M_{\infty}=\lim _{k \rightarrow \infty} M_{j_{k}}$, taken in $\Omega_{\infty} \times \mathbb{R}$ in the varifold sense, exists, and we have

$$
V_{\infty} \mathrm{L}\left(\Omega_{\infty} \times \mathbb{R}\right)=\mathbf{v}\left(M_{\infty}\right)
$$

and

$$
\mu_{r}\left(M_{\infty}\right)=M_{\infty} \quad \text { for } r>0 \quad \text { (that is, } \mu_{\infty} \text { is a cone). }
$$

(iii) $\mu_{\infty}$ is empty or $\mu_{\infty}=\bigcup_{j=1}^{N} \pi_{j} \cap\left(\Omega_{\infty} \times \mathbb{R}\right)$, where the $\pi_{j}$ are planes through the origin and $\pi_{i} \cap \pi_{j} \cap\left(\Omega_{\infty} \times \mathbb{R}\right)=\varnothing$ if $i \neq j$.

(iv) Either

Case 1. $N=1$ and $\mu_{\infty}=\pi_{1} \cap\left(\Omega_{\infty} \times \mathbb{R}\right)$ for some plane $\pi_{1}$ whose intersection with $\{O\} \times \mathbb{R}$ is $\left\{O_{3}\right\}$; or

Case 2. $N<\infty$ and $\mu_{\infty}=\bigcup_{j=1}^{N} \pi_{j} \cap\left(\Omega_{\infty} \times \mathbb{R}\right)$, where $\pi_{1}, \ldots, \pi_{N}$ are planes with the line $\{O\} \times \mathbb{R}$ in common.

(v) The subgraphs $U_{j_{k}}$ of $u_{j_{k}}$ and $U_{\infty}=\lim _{k \rightarrow \infty} \mu_{1 / \epsilon_{j_{k}}}(U)$ minimize appropriate functionals (for example, $\left[\mathrm{S},(3.4)^{\prime}\right]$ ).

Using (19) and arguing as in the proof of [S, (3.7), pages 373-4], we see that

$$
\mu_{\infty} \neq \varnothing \quad \text { and } \quad V_{\infty}=\mathbf{v}\left(\mu_{\infty}\right)
$$


(We note that this argument, specifically in the paragraph after [S, (3.7)], implies $\Theta^{* 1}\left(\|\delta V\|, O_{3}\right)<\infty$ and allows (15) to be used.) In particular, spt $\left(\left\|V_{\infty}\right\|\right)=\mathcal{M}_{\infty}$ and so (i) says that for each $\rho>0$, there is a sequence $\left\{\delta_{k}\right\}$ of positive reals that converges to zero such that

$$
B^{3}\left(O_{3}, \rho\right) \cap M_{j_{k}} \subset\left\{Y \in B^{3}\left(O_{3}, \rho\right): \operatorname{dist}\left(Y, M_{\infty}\right)<\delta_{k}\right\} .
$$

The conclusions in [S, Sections 2 and 3 up to, but not including, the paragraph containing (3.17)] hold and imply that $N=1, \mu_{\infty}=\pi_{1} \cap\left(\Omega_{\infty} \times \mathbb{R}\right)$ and either

- $\pi_{1} \cap(\{O\} \times \mathbb{R})=\left\{O_{3}\right\}$, or

- $\{O\} \times \mathbb{R} \subset \pi_{1}$.

(See also [Jeffres and Lancaster 2007].) We observe that the first is impossible when $\left(\gamma_{1}, \gamma_{2}\right) \in D_{2}^{+}\left(\right.$or $\left.\left(\gamma_{1}, \gamma_{2}\right) \in D_{2}^{-}\right)$since no plane can meet $\partial^{+} \Omega_{\infty} \times \mathbb{R}$ in angle $\gamma_{1}$ and $\partial^{-} \Omega_{\infty} \times \mathbb{R}$ in angle $\gamma_{2}$ (as Concus and Finn [1996] observed and an easy calculation confirms). Therefore there exists $\xi_{1}, \xi_{2} \in \mathbb{R}$ with $\xi_{1}^{2}+\xi_{2}^{2}=1$ and $\xi=\left(\xi_{1}, \xi_{2}, 0\right) \in S^{2}$ such that

$$
\pi_{1}=\left\{X \in \mathbb{R}^{3}: X \cdot \xi=0\right\} \quad \text { and } \quad U_{\infty}=\left\{X \in \Omega_{\infty} \times \mathbb{R}: X \cdot \xi>0\right\} .
$$

Hence

$$
\mu_{\infty}=\left\{X \in \mathbb{R}^{3}: X \cdot \xi=0\right\} \cap\left(\Omega_{\infty} \times \mathbb{R}\right)
$$

and we may write $U_{\infty}=U_{\infty}^{(1)} \times \mathbb{R}$, where $U_{\infty}^{(1)}=\left\{x \in \Omega_{\infty}: \xi \cdot(x, 0)>0\right\}$. Using the arguments in [S, pages 374-5], which yield [S, (3.13), (3.15)-(3.16) and (3.18)-(3.18)'] and, for example, defining

$$
\begin{array}{r}
E_{\infty}^{(1)}(W)=\mathscr{H}^{1}\left(\partial W \cap \Omega_{\infty} \cap B(O, 1)\right)-\cos \left(\gamma_{1}\right) \mathscr{H}^{1}\left(\partial W \cap \partial^{+} \Omega_{\infty} \cap B(O, 1)\right) \\
-\cos \left(\gamma_{2}\right) \mathscr{H}^{1}\left(\partial W \cap \partial^{-} \Omega_{\infty} \cap B(O, 1)\right)
\end{array}
$$

for any open set $W \subset \Omega_{\infty}$ satisfying

$$
\mathscr{H}^{1}(\partial W \cap B(O, 1))<\infty \quad \text { and } \quad\left(W \triangle U_{\infty}^{(1)}\right) \cap B(O, 1) \subset \subset B(O, 1),
$$

we obtain

$$
E_{\infty}^{(1)}\left(U_{\infty}^{(1)}\right) \leq E_{\infty}^{(1)}(W)
$$

for any set $W$ as described above (compare with [S, (3.16)].)

Note that $f_{j} \in B V\left(\Omega_{j}\right)$ is a variational solution and hence a generalized solution of (1) and (2) with $\Omega, \gamma$ and $H(x, z)$ replaced by $\Omega_{j}, \gamma_{j}$ and $H_{j}^{*}(x)=\epsilon_{j} H^{*}\left(\epsilon_{j} x\right)$ (with $H^{*}$ as in (46)) respectively. By Lemma 3.2, $\left(f_{j_{k}}\right)$ has a subsequence, still 
denoted $\left(f_{j_{k}}\right)$, that converges to a generalized solution $f_{\infty}: \Omega_{\infty} \rightarrow[-\infty, \infty]$ of

$$
\begin{aligned}
N v & =0 & & \text { in } \Omega_{\infty}, \\
T v \cdot v_{\infty}^{+} & =\cos \left(\gamma_{1}\right) & & \text { almost everywhere on } \partial^{+} \Omega_{\infty}, \\
T v \cdot v_{\infty}^{-} & =\cos \left(\gamma_{2}\right) & & \text { almost everywhere on } \partial^{-} \Omega_{\infty},
\end{aligned}
$$

where $v_{\infty}^{+}=\left(\cos \left(\alpha+\frac{1}{2} \pi\right), \sin \left(\alpha+\frac{1}{2} \pi\right)\right)$ and $\nu_{\infty}^{-}=\left(\cos \left(-\alpha-\frac{1}{2} \pi\right), \sin \left(-\alpha-\frac{1}{2} \pi\right)\right)$. Let us denote the subgraph of $f_{j}$ by

$$
U_{j}^{*}=\left\{(x, t) \in \Omega_{j} \times \mathbb{R}: t<f_{j}(x)\right\} \text { for } j \in \mathbb{N},
$$

and denote by $U_{\infty}^{*}$ the subgraph of $f_{\infty}$. Notice that (16) and $f_{j}\left(x_{j} /\left|x_{j}\right|\right)=0$ for $j \in \mathbb{N}$ imply

(a) if $K$ is open with $K \subset \subset \Omega_{\infty}$ and $(\cos (\beta), \sin (\beta)) \in K$, then there exists $m(K) \in \mathbb{N}$ such that $K \subset \Omega_{j}$ and $x_{j} /\left|x_{j}\right| \in K$ whenever $j \geq m(K)$;

(b) $\left(x_{j} /\left|x_{j}\right|, 0\right) \in \partial U_{j}^{*}$ for $j \in \mathbb{N}$; and

(c) $\left(x_{j} /\left|x_{j}\right|, 0\right) \rightarrow(\cos (\beta), \sin (\beta), 0)$ as $j \rightarrow \infty$.

Set $x_{\beta}=(\cos (\beta), \sin (\beta))$ and $X_{\beta}=(\cos (\beta), \sin (\beta), 0)$. From interior density bounds (for example, [Tam 1986b, Lemma 3.1]), we see that $X_{\beta} \in \partial U_{\infty}^{*} \cap\left(\Omega_{\infty} \times \mathbb{R}\right)$. Notice that $\mu^{*}=\partial U_{\infty}^{*} \cap\left(\Omega_{\infty} \times \mathbb{R}\right)$ is a smooth surface whose ("downward") unit normal can be denoted by $\vec{\chi}(X)=\left(\chi_{1}(X), \chi_{2}(X), \chi_{3}(X)\right)$ for $X \in \mathcal{M}^{*}$; then $\chi_{3}(X) \leq 0$ for all $X \in \mathcal{M}^{*}$. By Proposition 2.1, we see that

$$
\vec{n}_{j_{k}}\left(y_{k}\right) \rightarrow \vec{\chi}(X) \quad \text { as } k \rightarrow \infty \quad \text { whenever }\left(y_{k}, f_{j_{k}}\left(y_{k}\right)\right) \rightarrow X \in \mathcal{M}^{*} ;
$$

in particular, (c) implies $\vec{n}_{j_{k}}\left(x_{j_{k}} / \epsilon_{j_{k}}\right) \rightarrow \vec{\chi}\left(X_{\beta}\right)$ as $k \rightarrow \infty$ (with the set $\Omega$ in the proposition being a neighborhood of $X$ (or $\left.X_{\beta}\right)$ in $\mathbb{R}^{3}$.) We claim that either

(※) $\chi_{3}(X)<0$ for all $X \in \mathcal{M}^{*}$ or

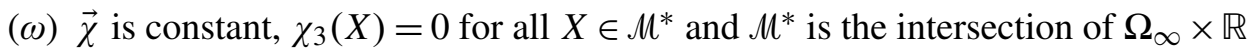
with the vertical plane $\pi_{2}$ containing $X_{\beta}$ and normal to $\vec{\chi}$.

(To see this, we may represent the minimal surface $M^{*}$ in isothermal coordinates as the (downward oriented) parametric surface $X: B(O, 1) \rightarrow \mathbb{R}^{3}$ (for example, [Courant 1977; Lancaster 1985; Elcrat and Lancaster 1986; Lancaster and Siegel 1996a]) and obtain the Weierstrass $(f, g)$-representation of $\mathcal{M}^{*}$, where

$$
g(w)=S(\vec{\chi}(X(u, v))) \quad \text { for } w=u+i v \in \mathbb{C},|w|<1,
$$

is the composition of the (north pole) stereographic projection $S$ with the Gauss map $\vec{\chi} \circ X: B(O, 1) \rightarrow S_{-}^{2}$. Then $g$ is a holomorphic map from the open unit ball in $\mathbb{C}$ into the closed unit ball in $\mathbb{C}$. If $\chi_{3}\left(X_{p}\right)=0$ for some $X_{p} \in \mathcal{M}^{*}$, then 
$X_{p}=X\left(u_{p}, v_{p}\right)$ for some $\left(u_{p}, v_{p}\right) \in B(O, 1)$ and $\left|g\left(w_{p}\right)\right|=1$ for $w_{p}=u_{p}+i v_{p}$; the maximum modulus principle implies $g$ is constant. The claim follows.)

Suppose ( $\aleph$ ) holds and $\chi_{3}(X)<0$ for all $X \in \mathcal{M}^{*}$. Then $f_{\infty} \in C^{2}\left(\Omega_{\infty}\right)$ and $\mathcal{M}^{*}$ is the graph of $f_{\infty}$ over $\Omega_{\infty}$. Let $0<R<\bar{R}<\operatorname{dist}\left(x_{\beta}, \partial \Omega_{\infty}\right)$; then there exists $L \geq 0$ such that $\left|\nabla f_{\infty}(x)\right| \leq L$ for all $x \in \overline{B\left(x_{\beta}, R\right)}$. Now (27) together with the uniform interior Hölder estimates for the unit normal (or Gauss) map of the graphs of solutions of (1) (for example, [Gilbarg and Trudinger 1983, Theorem 16.18] with $K=-1$ and $K^{\prime}=J(\bar{R}-R)^{2}$ or [S, (3.1)]) imply there exists $K(R, \bar{R})$ such that if $k \in \mathbb{N}$ satisfies $k \geq K(R, \bar{R})$, then $\overline{B\left(x_{\beta}, \bar{R}\right)} \subset \Omega_{j_{k}}$ and

$$
\left|\nabla u_{j_{k}}(x)\right| \leq L+1 \quad \text { for all } x \in \overline{B\left(x_{\beta}, R\right)} .
$$

Notice that for $k$ large enough, (28) contradicts (24) and (25) and so (※) cannot hold. Therefore $(\omega)$ holds, $M^{*}$ is the intersection of $\Omega_{\infty} \times \mathbb{R}$ with the plane $\pi_{2}$, we may write

$$
\vec{\chi}=(\cos (\theta), \sin (\theta), 0) \quad \text { for some } \theta \in(-\pi, \pi]
$$

and $U_{\infty}^{*}=\left\{X \in \Omega_{\infty} \times \mathbb{R}:\left(X-X_{\beta}\right) \cdot \vec{\chi}>0\right\}$. (Notice then that (24) and (25) imply $\vec{\chi}=\vec{\xi}$ and $\pi_{2}=\pi_{1}$.)

We will use the theory of generalized solutions (for example, [Giusti 1980]) to determine $\theta$. We claim that

$$
\theta= \begin{cases}-\alpha+\pi-\gamma_{2}+\pi / 2 & \text { if } \beta \in\left(-\alpha,-\alpha+\pi-\gamma_{2}\right], \\ \beta+\pi / 2 & \text { if } \beta \in\left[-\alpha+\pi-\gamma_{2}, \alpha-\gamma_{1}\right], \\ \alpha-\gamma_{1}+\pi / 2 & \text { if } \beta \in\left[\alpha-\gamma_{1}, \alpha\right) .\end{cases}
$$

The sets

$$
\mathscr{P}=\left\{x \in \Omega_{\infty}: f_{\infty}=\infty\right\} \quad \text { and } \quad \mathcal{N}=\left\{x \in \Omega_{\infty}: f_{\infty}=-\infty\right\}
$$

each minimize an appropriate functional, and the arguments in $[\mathrm{JL}]^{2}$ show that $U_{\infty}=\mathscr{P} \times \mathbb{R}$, where $\mathscr{P}$ is given in one of [JL, (iv), (vi) or (viii) of Theorem 1] and $\mathcal{N}=\Omega_{\infty} \backslash \overline{\mathscr{P}}$.

Suppose $\beta \in\left[-\alpha+\pi-\gamma_{2}, \alpha-\gamma_{1}\right]$ holds. We see that [JL, Theorem 1, case (viii)] must hold. Since $\partial \mathscr{P}$ is a line going through $O$ and $(\cos (\beta), \sin (\beta))$, we have $\vec{\chi}=(-\sin (\beta), \cos (\beta), 0)$ and $\theta=\beta+\pi / 2$.

Suppose $\beta \in\left(-\alpha,-\alpha+\pi-\gamma_{2}\right.$ ] holds. Then [JL, Theorem 1, case (vi)] must hold, $\vec{\chi}=\left(-\sin \left(-\alpha+\pi-\gamma_{2}\right), \cos \left(-\alpha+\pi-\gamma_{2}\right), 0\right)$ and $\theta=-\alpha+\pi-\gamma_{2}+\pi / 2$.

Finally, suppose $\beta \in\left[\alpha-\gamma_{1}, \alpha\right)$. Then [JL, Theorem 1, case (iv)] must hold, $\vec{\chi}=\left(-\sin \left(\alpha-\gamma_{1}\right), \cos \left(\alpha-\gamma_{1}\right), 0\right)$ and $\theta=\alpha-\gamma_{1}+\pi / 2$. Our claim (29) is therefore proven.

\footnotetext{
${ }^{2}$ Here [JL] stands for [Jeffres and Lancaster 2007].
} 
We have taken an arbitrary sequence $\left(x_{j}\right)$ in $\Omega$ that satisfies (16) and shown that it has a subsequence $\left(x_{j_{k}}\right)$ for which $\left(\vec{n}\left(x_{j_{k}}\right)\right)$ converges to $\vec{\chi}=(\cos (\theta), \sin (\theta), 0)$ with $\theta$ given by (29). Therefore, if $\left(y_{j}\right)$ is any sequence in $\Omega$ that satisfies (16) such that $\lim _{j \rightarrow \infty} \vec{n}\left(y_{j}\right)=\vec{\lambda}$ for some $S^{2} \ni \vec{\lambda} \neq \vec{\chi}$, it must have a subsequence $\left(y_{j_{k}}\right)$ for which $\vec{n}\left(y_{j_{k}}\right)$ converges to both $\vec{\lambda}$ and $\vec{\chi}$, which is a contradiction. Thus, we see that the conclusion of Theorem 2.1 follows.

Remark 2.2. Notice that $\left(\gamma_{1}, \gamma_{2}\right) \in D_{2}^{-}$if and only if $\left(\gamma_{2}, \gamma_{1}\right) \in D_{2}^{+}$and therefore we see that the conclusion of an appropriate version of Theorem 2.1 for the situation where $\left(\gamma_{1}, \gamma_{2}\right) \in D_{2}^{-}$follows using a reflection in the $x$-axis and Theorem 2.1.

Remark 2.3. The proof of [Tam 1986c, Section 1] is essentially the same as that used in [Simon 1980] with the modification that [S, (1.12)] does not hold, the twodimensional density $\Theta^{2}\left(\|Z\|, O_{3}\right)=0$ and $\mu_{\infty}=\varnothing$. Unfortunately the proofs of the claim in [Tam 1986c, Section 2] that (i) a subsequence of $\left\{f_{j}\right\}$ (called $\left\{u_{j}\right\}$ therein) converges "locally to a generalized solution" $f_{\infty}$ (called therein $u_{\infty}$ ) (that is, $\phi_{U_{j}^{*}} \rightarrow \phi_{U_{\infty}^{*}}$ in $L_{\text {loc }}^{1}\left(\Omega_{\infty} \times \mathbb{R}\right)$ with $U_{j}^{*}$ given by (26)) and (ii) the "graph" of this generalized solution (that is, $\partial U_{\infty}^{*}$ ) is a vertical plane are absent; the "blow-up" in that section does not correspond to the process of blowing up with respect to a fixed point (that is, $O_{3}$ ) used in [Simon 1980]. (In spite of this, the ideas in [Tam 1986c, Section 2] are remarkable.) One difficulty is that even if a subsequence of $\left\{f_{j}\right\}$ should happen to converge in the sense of [Giusti 1980] to a generalized solution $h_{\infty}$, the technique used here (for example, (24), (25), (28)) to show that $\partial U_{\infty}^{*}$ is a vertical plane cannot be used in [Tam 1986c] to show that $h_{\infty}$ is the generalized solution $u_{\infty}$ illustrated in [Tam 1986c, Figure 2 (see page 478)]. Even if Tam's proof can be correctly completed, the details would be sufficiently nontrivial that they should be provided to the reader. This new proof might be somewhat similar in outline to that of Theorem 2.1 above. (Of course, if $\alpha+\gamma<\pi / 2$ in [Tam 1986c], no such proof could exist; the potential correction would need to be cognizant of this fact.)

Remark 2.4. In some uses of geometric measure theory in the literature (for example, [Allard 1972; Taylor 1977]), the authors leave important details to the reader or adopt a glib, hand waving, style. In this style, the proof of Theorem 2.1 can be shortened to the following:

Proof sketch. Suppose $\left(x_{j}\right)$ is a sequence in $\Omega$ converging to $O$ as $j \rightarrow \infty$ and satisfying (16). For each $j \in \mathbb{N}$, set $\epsilon_{j}=\left|x_{j}\right|$ and $\Omega_{j}=\left\{x \in \mathbb{R}^{2}: \epsilon_{j} x \in \Omega\right\}$, and define $f_{j}, u_{j} \in C^{2}\left(\Omega_{j}\right) \cap C^{1}\left(\overline{\Omega_{j}} \backslash\{O\}\right)$ by

$$
f_{j}(x)=\frac{f\left(\epsilon_{j} x\right)-f\left(x_{j}\right)}{\epsilon_{j}} \quad \text { and } \quad u_{j}(x)=\frac{f\left(\epsilon_{j} x\right)-R f(\beta)}{\epsilon_{j}} .
$$


Using the techniques and results in [Simon 1980], we see that there is a vertical plane $\pi_{1}$ containing $O_{3}$ with unit normal $\vec{\chi}$ such that for each $\rho>0$, there is a sequence $\left\{\delta_{k}\right\}$ of positive reals that converges to zero such that

$$
B^{3}\left(O_{3}, \rho\right) \cap M_{j_{k}} \subset\left\{Y \in B^{3}\left(O_{3}, \rho\right): \operatorname{dist}\left(Y, \pi_{1}\right)<\delta_{k}\right\},
$$

where $\mu_{j}=\mu_{1 / \epsilon_{j}} \mu$ for each $k \in \mathbb{N}$; recall that $\mu_{r}(X)=r X, X \in \mathbb{R}^{3}$, and $\mu=$ $\left\{(x, f(x)-R f(\beta)): x \in \overline{\Omega \cap B\left(O, 3 \delta^{*}\right)} \backslash\{O\}\right\}$.

Now the sequence $\left\{f_{j}\right\}$ has a subsequence that converges (as in [Giusti 1980]) to a generalized solution $f_{\infty}$ of (1) and (2) (this is Lemma 3.2). Since $f_{j}\left(x_{j}\right)=0$, we have $\left(x_{j}, 0\right) \in \partial U_{j}^{*}$ for each $j \in \mathbb{N}$, where $U_{j}^{*}=\left\{(x, t) \in \Omega_{j} \times \mathbb{R}: t<f_{j}(x)\right\}$. Interior density bounds (for example, [Tam 1986b, Lemma 3.1]) imply

$$
(\cos (\beta), \sin (\beta), 0) \in \partial U_{\infty}^{*} \cap\left(\Omega_{\infty} \times \mathbb{R}\right),
$$

where $U_{\infty}^{*}$ is the subgraph of $f_{\infty}$. If $\partial U_{\infty}^{*}$ is not a vertical plane, then [Massari and Pepe 1975, Theorem 3] (that is, Proposition 2.1) and [Gilbarg and Trudinger 1983, Theorem 16.18] imply a uniform bound on $\left|\nabla f_{j}\right|$ in a neighborhood of $(\cos (\beta), \sin (\beta))$ in $\Omega_{\infty}$, and this contradicts (31) since $\nabla u_{j}=\nabla f_{j}$ for each $j \in \mathbb{N}$. The conclusions of Theorem 2.1 now follow from [Jeffres and Lancaster 2007, Theorem 1].

\section{Proof of Theorem 1.1}

The proof of this theorem uses the conformal (or isothermal) representation of a prescribed mean curvature surface discussed in [Lancaster and Siegel 1996a] and properties of two-dimensional quasiconformal maps to obtain a contradiction to the assumption that the solution $f$ is continuous at the origin. This proof uses Kenmotsu's theorem [1979], Theorem 2.1, Gehring's lemma [1973] and properties of solutions of Riemann-Hilbert problems to obtain a contradiction, illustrated in Figure 3, of the Phragmén-Lindelof theorem.

Proof. By Remark 2.2, we may assume $\left(\gamma_{1}, \gamma_{2}\right) \in D_{2}^{+}$. Assume $f$ is continuous at $O$; then $f$ is bounded in a neighborhood of $O$. Fix $\theta_{1} \in\left(-\alpha,-\alpha+\pi-\gamma_{2}\right)$ and $\theta_{2} \in\left(\alpha-\gamma_{1}, \alpha\right)$. By making $\delta_{0}>0$ smaller if necessary, we may assume

$$
\Omega^{*}=\left\{(r \cos (\theta), r \sin (\theta)): 0<r<\delta_{0}, \theta_{1}<\theta<\theta_{2}\right\}
$$

is contained in $\Omega$. Let $\partial^{+} \Omega^{*}=\left\{\left(r \cos \left(\theta_{2}\right), r \sin \left(\theta_{2}\right)\right): 0 \leq r \leq \delta_{0}\right\}$ and define $\gamma_{0}^{+}: \partial^{+} \Omega^{*} \rightarrow[0, \pi]$ so that $\cos \left(\gamma_{0}^{+}(x, y)\right)=T f(x, y) \cdot\left(\cos \left(\theta_{2}+\frac{1}{2} \pi\right), \sin \left(\theta_{2}+\frac{1}{2} \pi\right)\right)$ and notice that Theorem 2.1(iii) implies

$$
\gamma_{0}^{+}(x, y) \rightarrow \gamma_{1}+\theta_{2}-\alpha \quad \text { as }(x, y) \in \partial^{+} \Omega^{*} \text { goes to }(0,0) \text {. }
$$


Let $\partial^{-} \Omega^{*}=\left\{\left(r \cos \left(\theta_{1}\right), r \sin \left(\theta_{1}\right)\right): 0 \leq r \leq \delta_{0}\right\}$ and define $\gamma_{0}^{-}: \partial^{-} \Omega^{*} \rightarrow[0, \pi]$ so that $\cos \left(\gamma_{0}^{-}(x, y)\right)=T f(x, y) \cdot\left(\cos \left(\theta_{1}-\frac{1}{2} \pi\right), \sin \left(\theta_{1}-\frac{1}{2} \pi\right)\right)$ and notice that Theorem 2.1(ii) implies

$$
\gamma_{0}^{-}(x, y) \rightarrow \alpha+\gamma_{2}+\theta_{1} \quad \text { as }(x, y) \in \partial^{-} \Omega^{*} \text { goes to }(0,0) .
$$

Set

$$
\Pi=\left\{\left(\cos \left(\beta+\frac{1}{2} \pi\right), \sin \left(\beta+\frac{1}{2} \pi\right), 0\right): \pi-\alpha-\gamma_{2} \leq \beta \leq \alpha-\gamma_{1}\right\}
$$

and, for $s \in\left(0, \delta_{0}\right]$, let $\Omega_{s}=\left\{(x, y) \in \Omega^{*}: x^{2}+y^{2}<s^{2}\right\}$; notice that Theorem 2.1 implies

$$
\cap_{s>0} \overline{\vec{n}\left(\Omega_{s}\right)}=\Pi \text {. }
$$

Since $\alpha \leq \frac{1}{2} \pi$ and $\gamma_{2}-\gamma_{1}>\pi-2 \alpha$, we have $0<\frac{3}{2} \pi-\alpha-\gamma_{2}<\frac{1}{2} \pi+\alpha-\gamma_{1}<\pi$.

We now wish to examine the stereographic projection of the Gauss map near $(0,0, f(0,0))$ and represent it as the sum of a holomorphic function and a continuous function (that is, (44)).

From (32), (33) and (34) and the fact that $\gamma_{1}, \gamma_{2} \in(0, \pi)$, we see that there exists $\sigma \in\left(0, \delta_{0}\right]$ small enough that

$$
\vec{n}\left(\Omega_{\sigma}\right) \subset\left\{\omega(\theta, \phi): \frac{1}{4}\left(3 \pi-2 \alpha-2 \gamma_{2}\right)<\theta<\frac{1}{4}\left(3 \pi+2 \alpha-2 \gamma_{1}\right), \frac{1}{2} \pi<\phi<\frac{3}{4} \pi\right\},
$$

where $\omega(\theta, \phi)=(\sin (\phi) \cos (\theta), \sin (\phi) \sin (\theta), \cos (\phi))$, and there exists $\lambda>0$ such that $\lambda<\gamma_{0}^{ \pm}(x)<\pi-\lambda$ for $x \in \partial \Omega^{*} \backslash\{O\}$ with $|x| \leq \sigma$. Notice that $f \in C^{0}\left(\overline{\Omega_{\sigma}}\right) \cap$ $C^{2}\left(\overline{\Omega_{\sigma}} \backslash\{O\}\right)$ and that $f$ satisfies $N f=H(x, f)$ on $\Omega_{\sigma}, T f \cdot \boldsymbol{v}=\cos \left(\gamma_{0}^{+}\right)$on $\partial^{+} \Omega_{\sigma}=\Omega_{\sigma} \cap \partial^{+} \Omega^{*}$ and $T f \cdot \boldsymbol{v}=\cos \left(\gamma_{0}^{-}\right)$on $\partial^{-} \Omega_{\sigma}=\Omega_{\sigma} \cap \partial^{-} \Omega^{*}$. Define

$S_{0}=\left\{(x, y, f(x, y)):(x, y) \in \Omega_{\sigma}\right\} \quad$ and $\quad \Gamma_{0}=\left\{(x, y, f(x, y)):(x, y) \in \partial \Omega_{\sigma}\right\}$.

If $\Gamma_{0}^{ \pm}=\left\{(x, y, f(x, y)):(x, y) \in \partial^{ \pm} \Omega_{\sigma}, x^{2}+y^{2}<\sigma^{2}\right\}$ and $\Gamma_{0}^{\sigma}=\Gamma_{0} \backslash\left(\Gamma_{0}^{+} \cup \Gamma_{0}^{-}\right)$, then $\Gamma_{0}=\Gamma_{0}^{+} \cup \Gamma_{0}^{-} \cup \Gamma_{0}^{\sigma}$.

We will use the unit disk $E=\left\{(u, v): u^{2}+v^{2}<1\right\}$ as a parameter domain. From step 1 of the proof of [Lancaster and Siegel 1996a, Theorem 1] and from [Kenmotsu 1979] (also [Kenmotsu 2003]), we obtain the following facts.

There is a parametric description of the surface $S_{0}$

$$
X(u, v)=(x(u, v), y(u, v), z(u, v)) \in C^{2}\left(E: \mathbb{R}^{3}\right) \cap W^{1,2}\left(E: \mathbb{R}^{3}\right)
$$

with the following properties:

(i) $X$ is a homeomorphism of $E$ onto $S_{0}$.

(ii) $X$ maps $\partial E$ strictly monotonically onto $\Gamma_{0}$.

(iii) $X$ is conformal on $E$, that is, $X_{u} \cdot X_{v}=0$ and $\left|X_{u}\right|=\left|X_{v}\right|$ on $E$. 
(iv) Let $\tilde{H}(u, v)=H(X(u, v))$ denote the prescribed mean curvature of $S_{0}$ at $X(u, v)$. Then $\triangle X:=X_{u u}+X_{v v}=\tilde{H} X_{u} \times X_{v}$.

(v) $X \in C^{0}(\bar{E})$ and $X(1,0)=\left(0,0, z_{0}\right)$, where $z_{0}=f(0,0)$.

(vi) Write $G(u, v)=(x(u, v), y(u, v))$. Then $G(\cos t, \sin t)$ moves clockwise about $\partial \Omega_{\sigma}$ as $t$ increases in $0 \leq t \leq 2 \pi$, and $G$ is an orientation-reversing homeomorphism from $\bar{E}$ onto $\overline{\Omega_{\sigma}}$.

(vii) [Kenmotsu 1979, Lemma 1 and Corollary] Let $\pi_{S}: S^{2} \rightarrow \mathbb{C}$ denote the stereographic projection from the north pole and define $g(u+i v)=\pi_{S}(\vec{n}(G(u, v)))$ for $(u, v) \in E$. Then

$$
\left|g_{\bar{\zeta}}\right|=\frac{1}{2}|\tilde{H}|\left(1+|g|^{2}\right)\left|X_{u}\right|,
$$

where $\zeta=u+i v$,

$$
\frac{\partial}{\partial \zeta}=\frac{1}{2}\left(\frac{\partial}{\partial u}-i \frac{\partial}{\partial v}\right) \quad \text { and } \quad \frac{\partial}{\partial \bar{\zeta}}=\frac{1}{2}\left(\frac{\partial}{\partial u}+i \frac{\partial}{\partial v}\right) .
$$

For convenience with complex variables, set $E_{1}=\{\zeta \in \mathbb{C}:|\zeta|<1\}$.

Now Theorem 2.1 implies

$$
g(1+)=\lim _{\theta \downarrow 0} g\left(e^{i \theta}\right)=\cos \left(\alpha-\gamma_{1}+\frac{1}{2} \pi\right)+i \sin \left(\alpha-\gamma_{1}+\frac{1}{2} \pi\right)
$$

and

$$
g(1-)=\lim _{\theta \uparrow 0} g\left(e^{i \theta}\right)=\cos \left(\frac{3}{2} \pi-\alpha-\gamma_{2}\right)+i \sin \left(\frac{3}{2} \pi-\alpha-\gamma_{2}\right) .
$$

Define $\tilde{n}(u, v)=\vec{n}(x(u, v), y(u, v))$ for $(u, v) \in E$. Notice that if

$$
\tilde{n}(u, v)=\left(\tilde{n}_{1}(u, v), \tilde{n}_{2}(u, v), \tilde{n}_{3}(u, v)\right),
$$

then, from the choice of $\sigma$,

$$
-\cot \left(\frac{1}{4} \pi+\frac{1}{2}\left(\alpha-\gamma_{1}\right)\right)<\frac{\tilde{n}_{1}(u, v)}{\tilde{n}_{2}(u, v)}<\cot \left(\frac{3}{4} \pi-\frac{1}{2}\left(\alpha-\gamma_{2}\right)\right)
$$

and

(38) $\min \left\{-\csc \left(\frac{1}{4}\left(3 \pi-2 \alpha-2 \gamma_{2}\right)\right),-\csc \left(\frac{1}{4}\left(3 \pi+2 \alpha-2 \gamma_{1}\right)\right)\right\}<\frac{\tilde{n}_{3}(u, v)}{\tilde{n}_{2}(u, v)}<0$.

Now

$$
\tilde{n}(u, v)=\frac{X_{u} \times X_{v}}{\left|X_{u} \times X_{v}\right|}=\frac{1}{\left|X_{u}\right|^{2}}\left(y_{u} z_{v}-y_{v} z_{u}, x_{v} z_{u}-x_{u} z_{v}, x_{u} y_{v}-x_{v} y_{u}\right) ;
$$

hence

$$
\frac{\left|x_{u} y_{v}-x_{v} y_{u}\right|}{\left|x_{v} z_{u}-x_{u} z_{v}\right|}=\frac{\left|\tilde{n}_{3}\right|}{\left|\tilde{n}_{2}\right|}<A \quad \text { and } \quad \frac{\left|y_{u} z_{v}-y_{v} z_{u}\right|}{\left|x_{v} z_{u}-x_{u} z_{v}\right|}=\frac{\left|\tilde{n}_{1}\right|}{\left|\tilde{n}_{2}\right|}<B
$$


where

$$
\begin{aligned}
& A=\max \left\{\csc \left(\frac{1}{4}\left(3 \pi-2 \alpha-2 \gamma_{2}\right)\right), \csc \left(\frac{1}{4}\left(3 \pi+2 \alpha-2 \gamma_{1}\right)\right)\right\}, \\
& B=\max \left\{\cot \left(\frac{1}{4} \pi+\frac{1}{2}\left(\alpha-\gamma_{1}\right)\right), \cot \left(\frac{3}{4} \pi-\frac{1}{2}\left(\alpha-\gamma_{2}\right)\right)\right\} .
\end{aligned}
$$

Now $(\partial f / \partial y)(x(u, v), y(u, v))=-\tilde{n}_{2}(u, v) / \tilde{n}_{3}(u, v)$ and so (38) implies

$$
\frac{\partial f}{\partial y} \geq \min \left\{\sin \left(\frac{1}{4}\left(3 \pi-2 \alpha-2 \gamma_{2}\right)\right), \sin \left(\frac{1}{4}\left(3 \pi+2 \alpha-2 \gamma_{1}\right)\right)\right\}>0
$$

and so $S_{0}=\left\{(x, y, f(x, y)):(x, y) \in \Omega_{\sigma}\right\}=X(E)$ is the graph $y=\phi(z, x)$ over the $(z, x)$-plane of a $C^{2}$ function over the projection $U$ of $\mathscr{S}_{0}$ on the $(z, x)$-plane. Notice that $\phi \in C^{0}(\bar{U})$ and $\partial U$ is the projection of $\Gamma_{0}$ on the $(z, x)$-plane.

If $\partial_{0} U=\left\{(z, x):(x, y, z) \in \Gamma_{0}^{+} \cup \Gamma_{0}^{-}\right\}$and $\partial_{1} U=\left\{(z, x):(x, y, z) \in \Gamma_{0}^{\sigma}\right\}$, then $\partial U=\partial_{0} U \cup \partial_{1} U$. Now Theorem 2.1 implies $|\nabla f(x, y)| \rightarrow \infty$ as $(x, y) \in \Omega^{*}$ goes to $O$. Also

$$
T f\left(r \cos \left(\theta_{2}\right), r \sin \left(\theta_{2}\right)\right) \cdot\left(\cos \left(\theta_{2}\right), \sin \left(\theta_{2}\right)\right) \rightarrow \cos \left(\alpha+\frac{1}{2} \pi-\gamma_{1}-\theta_{2}\right)>0,
$$
since $\frac{1}{2} \pi-\gamma_{1}<\alpha+\frac{1}{2} \pi-\gamma_{1}-\theta_{2}<\frac{1}{2} \pi$, and

$T f\left(r \cos \left(\theta_{1}\right), r \sin \left(\theta_{1}\right)\right) \cdot\left(\cos \left(\theta_{1}\right), \sin \left(\theta_{1}\right)\right) \rightarrow \cos \left(\frac{3}{2} \pi-\alpha-\gamma_{2}-\theta_{1}\right)<0$, since $\frac{1}{2} \pi<\frac{3}{2} \pi-\alpha-\gamma_{2}-\theta_{1}<\frac{3}{2} \pi-\gamma_{2}$. Thus the limits of the directional derivatives of $f$ in the directions of $\partial^{+} \Omega_{\sigma}$ and $\partial^{-} \Omega_{\sigma}$ are

$$
\begin{aligned}
& \lim _{r \downarrow 0} \nabla f\left(r \cos \left(\theta_{2}\right), r \sin \left(\theta_{2}\right)\right) \cdot\left(\cos \left(\theta_{2}\right), \sin \left(\theta_{2}\right)\right)=+\infty, \\
& \lim _{r \downarrow 0} \nabla f\left(r \cos \left(\theta_{1}\right), r \sin \left(\theta_{1}\right)\right) \cdot\left(\cos \left(\theta_{1}\right), \sin \left(\theta_{1}\right)\right)=-\infty .
\end{aligned}
$$

Hence $\Gamma_{0}^{+}$is tangent to $\left\{(0,0, z): z \geq z_{0}\right\}$ and $\Gamma_{0}^{-}$is tangent to $\left\{(0,0, z): z \leq z_{0}\right\}$ at $\left(0,0, z_{0}\right)$. In addition, $(\partial \phi / \partial z)\left(z_{0}, 0\right)=0$. Thus $\Gamma_{0}^{+} \cup \Gamma_{0}^{-}$is a $C^{1}$ curve and $\partial U$ is the union of the $C^{1}$ curve $\partial_{0} U$ and the $C^{2}$ curve $\partial_{1} U$. Since

$$
\left|\nabla f\left(\sigma \cos \left(\theta_{2}\right), \sigma \sin \left(\theta_{2}\right)\right)\right|<\infty,
$$

$f_{y}\left(\sigma \cos \left(\theta_{2}\right), \sigma \sin \left(\theta_{2}\right)\right)>0$ (by (40)) and the curves $y=\tan \left(\theta_{2}\right) x$ and $x^{2}+y^{2}=\sigma^{2}$ are orthogonal at $\left(\sigma \cos \left(\theta_{2}\right), \sigma \sin \left(\theta_{2}\right)\right)$, we see that $\Gamma_{0}^{+}$and $\Gamma_{0}^{\sigma}$ do not meet tangentially at $\left(\sigma \cos \left(\theta_{2}\right), \sigma \sin \left(\theta_{2}\right), f\left(\sigma \cos \left(\theta_{2}\right), \sigma \sin \left(\theta_{2}\right)\right)\right)$ and $\partial_{0}^{+} U$ and $\partial_{1} U$ do not meet tangentially at $\left(\sigma \cos \left(\theta_{2}\right), \sigma \sin \left(\theta_{2}\right)\right)$. Similarly $\Gamma_{0}^{-}$and $\Gamma_{0}^{\sigma}$ do not meet tangentially at $\left(\sigma \cos \left(\theta_{1}\right), \sigma \sin \left(\theta_{1}\right), f\left(\sigma \cos \left(\theta_{1}\right), \sigma \sin \left(\theta_{1}\right)\right)\right)$ and $\partial_{0}^{-} U$ and $\partial_{1} U$ do not meet tangentially at $\left(\sigma \cos \left(\theta_{1}\right), \sigma \sin \left(\theta_{1}\right)\right)$. Therefore $U$ is a simply connected Lipschitz domain and $\partial U$ is a quasicircle (see [Gehring 2005, Theorem 6.3]).

Let us define $F \in C^{2}\left(E: \mathbb{R}^{2}\right) \cap W^{1,2}\left(E: \mathbb{R}^{2}\right)$ by $F(u, v)=(z(u, v), x(u, v))$. Note that $F$ is a homeomorphism from $\bar{E}$ onto $\bar{U}$. Recall that $|D F|^{2}=x_{u}^{2}+x_{v}^{2}+z_{u}^{2}+z_{v}^{2}$ 
and the determinant of $D F$ at $(u, v)$ is

$$
J((u, v), F)=x_{v} z_{u}-x_{u} z_{v}=\left|X_{u}\right|^{2} \tilde{n}_{2}>0 .
$$

Since we are using conformal, or isothermal, coordinates, we obtain

$$
\begin{aligned}
|D F(u, v)|^{2} \leq 2\left|X_{u}\right|^{2} & =2\left|X_{u} \times X_{v}\right| \\
& =2 \sqrt{\left(y_{u} z_{v}-y_{v} z_{u}\right)^{2}+\left(x_{v} z_{u}-x_{u} z_{v}\right)^{2}+\left(x_{u} y_{v}-x_{v} y_{u}\right)^{2}} \\
& \leq 2 \sqrt{\left(B^{2}+1+A^{2}\right)\left(x_{v} z_{u}-x_{u} z_{v}\right)^{2}}=2 K J((u, v), F),
\end{aligned}
$$

where $K=\sqrt{B^{2}+1+A^{2}}$. Thus $F$ is a $K^{\prime}$-quasiconformal map from $E$ to $U$, where $K^{\prime}=\left(K-\sqrt{K^{2}-1}\right)^{-1}=\sqrt{A^{2}+B^{2}+1}+\sqrt{A^{2}+B^{2}}$; see for example [Finn and Serrin 1958]. Then [Gehring 2005, Theorem 6.4] implies that there is a $K^{\prime}$-quasiconformal extension $L: \mathbb{R}^{2} \rightarrow \mathbb{R}^{2}$ of $F^{-1}: \bar{U} \rightarrow \bar{E}$ and hence there is a $K^{\prime}$-quasiconformal extension $\tilde{F}: \mathbb{R}^{2} \rightarrow \mathbb{R}^{2}$ of $F$, given by $\tilde{F}=L^{-1}$. Using Gehring's lemma [1973] or [Iwaniec and Martin 2001, Theorem 14.4.1], we see that $\tilde{F} \in W^{1, p}(B((1,0), \delta))$ for some $p>2$. Since $\tilde{F}=F$ on $E \cap B((1,0), 2 \delta)$ and $F \in W^{1, \infty}(E \backslash B((1,0), \epsilon))$ for each $\epsilon>0$, we see that $x_{u}, x_{v}, z_{u}, z_{v} \in L^{p}(E)$. Since $\tilde{n}$ is normal to $X(E)$, we have $X_{u} \cdot \tilde{n}=0$ and $X_{v} \cdot \tilde{n}=0$, which imply

$$
y_{u}=\frac{\tilde{n}_{1}}{\tilde{n}_{2}} x_{u}+\frac{\tilde{n}_{3}}{\tilde{n}_{2}} z_{u} \quad \text { and } \quad y_{v}=\frac{\tilde{n}_{1}}{\tilde{n}_{2}} x_{v}+\frac{\tilde{n}_{3}}{\tilde{n}_{2}} z_{v}
$$

and therefore $\left|y_{u}\right| \leq B\left|x_{u}\right|+A\left|z_{u}\right|$ and $\left|y_{v}\right| \leq B\left|x_{v}\right|+A\left|z_{v}\right|$. This implies $X$ belongs to $W^{1, p}\left(E: \mathbb{R}^{3}\right)$.

The corollary on [Kenmotsu 1979, page 92] yields

$$
\left|g_{\bar{\zeta}}\right|=\frac{1}{2}|\tilde{H}|\left(1+|g|^{2}\right)\left|X_{u}\right| \leq|H|_{\infty}\left|X_{u}\right|
$$

and so $g_{\bar{\zeta}} \in L^{p}\left(E_{1}: \mathbb{R}^{2}\right)$. Let us set $\mu=(p-2) / p$. Then from [Monakhov 1983, Theorems 5 and 6 , page 205], we see that

$$
g(\zeta)=\psi(\zeta)+h(\zeta)
$$

where $\psi$ is a holomorphic function and $h \in L^{\infty}\left(E_{1}\right)$ is a uniformly Hölder continuous function on $E_{1}$ with Hölder exponent $\mu$. Since $g$ and $h$ are bounded, so is $\psi$. Since $h$ is continuous at $1 \in \partial E_{1}$ and $\psi(\zeta)=g(\zeta)-h(\zeta)$, the Phragmén-Lindelof theorem (for example, [Bear and Hile 1983]) and Theorem 2.1 imply

$$
\lim _{r \rightarrow 0^{+}} \psi(1+r \cos (\theta)+i r \sin (\theta))=\psi(1-)(\theta / \pi-1 / 2)+\psi(1+)(3 / 2-\theta / \pi)
$$

for $\pi / 2<\theta<3 \pi / 2$, where $\psi(1+)=g(1+)-h(1)$ and $\psi(1-)=g(1-)-h(1)$, and so

$$
\lim _{r \rightarrow 0^{+}} g(1+r \cos (\theta)+i r \sin (\theta))=g(1-)(\theta / \pi-1 / 2)+g(1+)(3 / 2-\theta / \pi)
$$




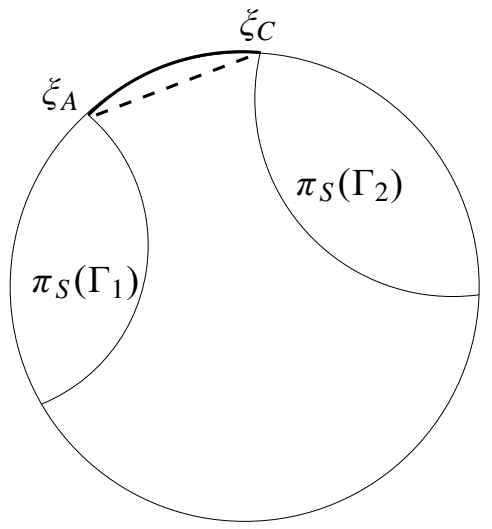

Figure 3. Differing limits of $g(u+i v)$ as $u+i v \rightarrow 1$.

for $\pi / 2<\theta<3 \pi / 2$. Notice then that along any ray $\{1+r \cos (\theta)+i r \sin (\theta)\}$ with $\pi / 2<\theta<3 \pi / 2$, in $|\zeta|<1, g$ converges to a point strictly inside the open unit disk as 1 is approached; see Figure 3. This contradicts Theorem 2.1, which implies $|g(u+i v)| \rightarrow 1$ as $u+i v \rightarrow 1$. Thus our assumption that $f$ is continuous at $O$ is invalid and the proof of Theorem 1.1 is complete.

Remark 3.1. In [Lancaster and Siegel 1996a, Theorem 1], the hypotheses include, "If $\alpha<\pi / 2$ and there exist constants $\underline{\gamma}^{ \pm}, \bar{\gamma}^{ \pm}, 0<\underline{\gamma}^{ \pm} \leq \pi / 2, \pi / 2 \leq \bar{\gamma}^{ \pm}<\pi$, satisfying

$$
\underline{\gamma}^{+}+\underline{\gamma}^{-}>\pi-2 \alpha \text { and } \bar{\gamma}^{+}+\bar{\gamma}^{-}<2 \alpha+\pi
$$

so that $\gamma^{ \pm} \leq \gamma^{ \pm}(s) \leq \bar{\gamma}^{ \pm}$for all $s$ in $0<s<s_{0}$ for some $s_{0}$."

While the theorem is true as stated, the assumptions $\underline{\gamma}^{ \pm} \leq \pi / 2$ and $\pi / 2 \leq \bar{\gamma}^{ \pm}$ were added as an afterthought (by this author) and were unnecessary to the argument; [Lancaster and Siegel 1996a, Theorems 1 and 2] remain correct if one merely assumes $\underline{\gamma}^{ \pm} \leq \bar{\gamma}^{ \pm}$. It is useful to note this fact because these assumptions artificially restrict the applicability of these theorems. (In fact, the remainder of that article correctly ignores this restriction.)

\section{Appendix}

We wish to discuss variational solutions of (1) and (2). We assume a solution $f \in C^{2}(\Omega) \cap C^{1, \rho^{*}}(\bar{\Omega} \backslash\{O\})$ is given and we define $H^{*}: \Omega \times \mathbb{R} \rightarrow \mathbb{R}$ by

$$
H^{*}(x)=H(x, f(x)) \quad \text { for } x \in \Omega .
$$

For the moment, we let $\Omega$ be any connected, open subset of $\mathbb{R}^{2}$ that has locally Lipschitz boundary and let $\gamma \in L^{\infty}(\partial \Omega)$ with $0 \leq \gamma(x, y) \leq \pi$ for $(x, y) \in \partial \Omega$; for convenience of notation, we assume $\Omega$ is bounded. The usual definition of a 
$B V(\Omega)$ (variational) solution of (1) and (2) is a function $u \in B V(\Omega)$ that minimizes the functional

$$
\mathscr{E}(u)=\int_{\Omega} \sqrt{1+|D u|^{2}} d H_{2}+\int_{\Omega} \int_{0}^{u} H^{*}(\cdot, t) d t d H_{2}-\int_{\partial \Omega} \cos (\gamma) u d H_{1}
$$

over $B V(\Omega)$. In some cases (for example, $\Omega$ is unbounded), individual terms in the functional may be infinite; Finn [1986, Definition 7.1] offers a more general definition of variational solution in his book. Another type of variational solution of (1) and (2) is that of a generalized solution, which we describe next.

We denote by $\mathscr{F}$ the (formal) functional given by

$$
\mathscr{F}(U)=\int_{\Omega \times \mathbb{R}}\left|D \phi_{U}\right|+\int_{\Omega \times \mathbb{R}} H^{*} \phi_{U} d t d H_{2}-\int_{\partial \Omega \times \mathbb{R}} \cos (\gamma) \phi_{U} d H_{2} .
$$

For each $T \in(0, \infty)$ and $K \subset \subset \mathbb{R}^{2}$, we define the functional

$$
\mathscr{F}_{T, K}(U)=\int_{\Omega(T, K)}\left|D \phi_{U}\right|+\int_{\Omega(T, K)} H^{*} \phi_{U} d t d H_{2}-\int_{\delta \Omega(T, K)} \cos (\gamma) \phi_{U} d H_{2}
$$

when $U \subset \Omega \times \mathbb{R}$ is a Caccioppoli set (that is, a Borel set with locally finite perimeter $)$, where $\Omega(T, K)=(\Omega \cap K) \times(-T, T)$ and $\delta \Omega(T, K)=(\partial \Omega \cap K) \times(-T, T)$.

Definition 3.1. A Caccioppoli set $U \subset \Omega \times \mathbb{R}$ is said to be a local solution for $\mathscr{F}$ if and only if for each $T>0$ and $K \subset \subset \mathbb{R}^{2}$, we have $\mathscr{F}_{T, K}(U) \leq \mathscr{F}_{T, K}(V)$ whenever $V \subset \Omega \times \mathbb{R}$ is a Caccioppoli set such that the support of $\phi_{U}-\phi_{V}$ is contained in $\Omega(T, K)$.

As noted in [Finn 1986, Section 7.3], a function $u \in B V(\Omega)$ minimizes $\mathscr{E}$ if and only if its subgraph $U=\{(x, y, t) \in \Omega \times \mathbb{R}: t<u(x, y)\}$ is a local solution for $\mathscr{F}$ [Miranda 1977].

Definition 3.2. A function $u: \Omega \rightarrow[-\infty, \infty]$ is called a generalized solution of (1) and (2) if and only if its subgraph $U$ is a local solution for $\mathscr{F}$.

Definition 3.3. A sequence $\left(u_{j}\right)$ in $B V(\Omega)$ is said to converge locally in $\Omega$ to $u_{\infty}$ if and only if $\phi_{U_{j}}$ converges to $\phi_{U_{\infty}}$ in $L_{\mathrm{loc}}^{1}(\Omega \times \mathbb{R})$ as $j \rightarrow \infty$, where $U_{j}$ and $U_{\infty}$ are the subgraphs of $u_{j}$ and $u_{\infty}$, respectively.

Definition 3.4. For each $\Lambda \subset \mathbb{R}^{2}$ and $\epsilon>0$, set $\Lambda^{\epsilon}=\Lambda \backslash B(O, \epsilon)$ and $\Sigma^{\epsilon}=$ $\partial \Lambda \backslash B(O, \epsilon)$. We will say the triple $(\lambda, \Lambda, O)$ is admissible if and only if $\Lambda$ is an open set in $\mathbb{R}^{2}, O \in \partial \Lambda$, the map $\lambda: \partial \Lambda \backslash\{O\} \rightarrow(0, \pi)$ is in $C^{0, \rho^{*}}(\partial \Lambda \backslash\{O\})$ and, for some $\epsilon_{0}>0$ and all $\epsilon \in\left(0, \epsilon_{0}\right]$, there exist $a=a(\epsilon) \in(0,1), \tau=\tau(\epsilon)>0$, $N=N(\epsilon), \quad N_{1}=N_{1}(\epsilon) \leq N(\epsilon)$, a finite open cover $\left\{\Lambda_{j}^{\epsilon}: j=2, \ldots, N\right\}$ of $\overline{\Omega^{\epsilon}}$ with $O \notin \bigcup_{j=2}^{N} \overline{\Lambda_{j}^{\epsilon}}$ and rigid motions $F_{j}: \mathbb{R}^{2} \rightarrow \mathbb{R}^{2}$ for $2 \leq j \leq N_{1}$, such that $\Lambda_{j}^{\epsilon} \cap \partial \Lambda \neq \varnothing$ if $1 \leq j \leq N_{1}$ and $\Lambda_{j}^{\epsilon} \cap \partial \Lambda=\varnothing$ if $N_{1}<j \leq N$, the set $\Sigma_{j}^{\epsilon}=\partial \Lambda \cap \Lambda_{j}^{\epsilon}$ is open and connected in the relative topology of $\Sigma^{\epsilon}, F_{j}\left(\Sigma_{j}^{\epsilon}\right)$ can be represented 
over some interval $a_{j}<x<b_{j}$ with $a_{j}<b_{j}$ by a Lipschitz function $y=\psi_{j}(x)$ with Lipschitz constant $L_{j}$, the set $T_{j}=\left\{\left(x, y+\psi_{j}(x)\right): a_{j}<x<b_{j},-\tau<y<0\right\}$ lies in $F_{j}(\Omega)$ and $|\cos (\gamma)| \sqrt{1+L_{j}^{2}} \leq a(\epsilon)$ on $\Sigma_{j}^{\epsilon}$ for $j=2, \ldots, N_{1}$. Compare this with [Finn 1986, Section 6.3].

Lemma 3.1. Suppose $\gamma \in C^{0, \rho^{*}}(\partial \Omega \backslash\{O\})$ satisfies (4), $\left|\gamma_{1}-\gamma_{2}\right|>\pi-2 \alpha$ (so that $\left.\left(\gamma_{1}, \gamma_{2}\right) \in D_{2}^{+} \cup D_{2}^{-}\right)$and $(\gamma, \Omega, O)$ is admissible. Then there exist $\zeta>0$, $\mu=\mu(a(\zeta), \Omega)$ and $\Upsilon=\Upsilon(a(\zeta), \Omega)$ with $\mu \in[a(\zeta), 1)$ such that for each $T>0$, $\lambda>0$ and $f \in B V(\Omega \times(-T, T))$ with $f \geq 0$ almost everywhere on $\Omega \times(-T, T)$, we have

$$
\left|\int_{\Sigma \times(-T, T)} \cos (\gamma) f^{*} d H_{2}\right| \leq \mu \int_{\mathscr{A}_{\lambda} \times(-T, T)}|D f|+\Upsilon \int_{\mathscr{A}_{\lambda} \times(-T, T)} f,
$$

where $A_{\lambda} \subset \Omega$ is the strip of width $\lambda$ adjacent to $\Sigma=\partial \Omega$ and we denote by $f^{*} \in L^{1}(\partial \Omega \times(-T, T))$ the trace of $f$ on $\partial \Omega \times(-T, T)$.

Proof. Fix $T>0$ and $\lambda>0$. Let $f \in B V(\Omega \times(-T, T))$ such that $f \geq 0$ almost everywhere in $\Omega \times(-T, T)$; then $f^{*} \geq 0$ almost everywhere on $\partial \Omega$. We see from [Giusti 1984, Remark 2.12] that there exists a sequence

$$
\left\{f_{k}\right\} \subset C^{\infty}(\Omega \times(-T, T)) \cap B V(\Omega \times(-T, T))
$$

such that

$$
\begin{aligned}
& \lim _{k \rightarrow \infty} \int_{\Omega \times(-T, T)}\left|f_{k}-f\right| d x=0, \\
& \lim _{k \rightarrow \infty} \int_{\Omega \times(-T, T)}\left|D f_{k}\right| d x=\int_{\Omega \times(-T, T)}|D f|
\end{aligned}
$$

and

$$
f_{k}^{*}=f^{*} \quad \text { on } \partial(\Omega \times(-T, T)) \text { for each } k \in \mathbb{N},
$$

where $f_{k}^{*}$ and $f^{*}$ denote the traces of $f_{k}$ and $f$ on $\partial(\Omega \times(-T, T))$, respectively. An examination of the construction of the $f_{k}$ in [Giusti 1984, Theorem 1.17] shows that $f_{k} \geq 0$ on $\Omega \times(-T, T)$ for $k=1,2,3, \ldots$, since $f \geq 0$ almost everywhere on $\Omega \times(-T, T)$. (In fact, each $f_{k}$ is actually a function $f_{\epsilon}$ for a suitably small $\epsilon>0$ in the construction in the proof of that theorem.)

Since $\int|D f|$ is a Radon measure on $\Omega \times(-T, T)$,

$$
\int_{\partial \mathscr{A}_{\sigma} \times(-T, T)}|D f|=0 \quad \text { for almost all } \sigma \in(0, \lambda] \text { and all } T>0 ;
$$

by replacing $\lambda$ by a $\sigma \in(0, \lambda]$ that satisfies (52), we may assume

$$
\int_{\partial \mathscr{A}_{\lambda} \times(-T, T)}|D f|=0
$$


always holds.

We shall focus on functions $h \in C^{1}(\Omega \times(-T, T)) \cap B V(\Omega \times(-T, T))$ with $h \geq 0$ in $\Omega \times(-T, T)$ ), obtain (48) for such functions, and then use the approximation above to establish (48) for $f$.

Case $1\left(\left(\gamma_{1}, \gamma_{2}\right) \in D_{2}^{+}\right.$and $\left.\gamma_{2} \leq \pi / 2\right)$. This case is defined by $\gamma_{2}-\gamma_{1}>\pi-2 \alpha$, and so $\gamma_{1}<\pi / 2$ and $2 \alpha>\pi / 2$. Fix $\epsilon \in\left(0, \gamma_{1}\right)$. We wish to select $\sigma \in(0, \pi / 2)$ such that $\sigma<\gamma_{1}-\epsilon, 0<\pi-2 \alpha-\sigma<\gamma_{2}-\epsilon$. Now these conditions require that $\sigma \in\left(0, \gamma_{1}-\epsilon\right) \cap\left(\pi-2 \alpha-\gamma_{2}+\epsilon, \pi-2 \alpha\right)$; this intersection is nonempty since $\gamma_{1}-\epsilon-\left(\pi-2 \alpha-\gamma_{2}+\epsilon\right)>2 \gamma_{1}-2 \epsilon>0$ and so $\gamma_{1}-\epsilon>\pi-2 \alpha-\gamma_{2}+\epsilon$.

Let $\zeta>0$ be small enough that

(a) $\left|\gamma(x)-\gamma_{1}\right|<\epsilon / 2$ whenever $x \in \partial^{+} \Omega \backslash\{O\}$ with $|x| \leq 2 \zeta$, and

(b) $\left|\gamma(x)-\gamma_{2}\right|<\epsilon / 2$ whenever $x \in \partial^{-} \Omega \backslash\{O\}$ with $|x| \leq 2 \zeta$.

Recall that $\tau^{+}(x)=\gamma(x)-\pi / 2$ for $x \in \partial^{+} \Omega \cap B_{\delta^{*}}(O)$ and $\tau^{-}(x)=\gamma(x)+\pi / 2$ for $x \in \partial^{-} \Omega \cap B_{\delta^{*}}(O)$; hence $\left|\tau^{+}(x)-\alpha\right|<\epsilon / 2$ whenever $x \in \partial^{+} \Omega$ with $|x| \leq 2 \zeta$ and $\left|\tau^{-}(x)+\alpha\right|<\epsilon / 2$ whenever $x \in \partial^{-} \Omega$ with $|x| \leq 2 \zeta$.

Let $\tau=\zeta$ and $R_{1}: \mathbb{R}^{2} \rightarrow \mathbb{R}^{2}$ be the rotation about the origin through the angle $-\alpha-\sigma$. Then $R_{1}\left(\partial^{+} \Omega\right)$ and $R_{1}\left(\partial^{-} \Omega\right)$ are the graphs $y=\psi_{1}^{+}(x)$ and $y=\psi_{1}^{-}(x)$ of Lipschitz functions with Lipschitz constants

$$
L_{1}^{+} \leq \tan (\sigma+\epsilon / 2) \quad \text { and } \quad L_{1}^{-} \leq \tan (\pi-2 \alpha-\sigma+\epsilon / 2),
$$

respectively; notice that $\operatorname{dom}\left(\psi_{1}^{+}\right)=\left[0, x_{0}^{+}\right)$and $\operatorname{dom}\left(\psi_{1}^{-}\right)=\left(x_{0}^{-}, 0\right]$, where

$$
\left|\left(x_{0}^{+}, \psi_{1}^{+}\left(x_{0}^{+}\right)\right)\right|=2 \zeta \quad \text { and } \quad\left|\left(x_{0}^{-}, \psi_{1}^{-}\left(x_{0}^{-}\right)\right)\right|=2 \zeta .
$$

Set $L_{1}=\max \left\{L_{1}^{+}, L_{1}^{-}\right\}$and let $\delta>0$ satisfy $\delta^{2}+\left(L_{1} \delta+\tau\right)^{2}=4 \zeta^{2}$ (so that $\left.\delta=\zeta\left(\left(3 L_{1}^{2}+4\right)^{1 / 2}-1\right) /\left(L_{1}^{2}+1\right)\right)$.

For $0<x \leq \delta$, we have $\sigma+\epsilon / 2<\gamma_{1}-\epsilon / 2<\gamma(x)$ and so

$$
\cos (\gamma(x)) \sqrt{1+\left(L_{1}^{+}\right)^{2}}<\cos \left(\gamma_{1}-\epsilon / 2\right) \sec (\sigma+\epsilon / 2)<\frac{\cos \left(\gamma_{1}-\epsilon / 2\right)}{\cos \left(\gamma_{1}-\epsilon / 2\right)}=1 .
$$

For $-\delta \leq x<0$, we have $\pi-2 \alpha-\sigma+\epsilon / 2<\gamma_{2}-\epsilon / 2<\gamma(x)$ and so $\cos (\gamma(x)) \sqrt{1+\left(L_{1}^{-}\right)^{2}}<\cos \left(\gamma_{2}-\epsilon / 2\right) \sec (\pi-2 \alpha-\sigma+\epsilon / 2)<\frac{\cos \left(\gamma_{2}-\epsilon / 2\right)}{\cos \left(\gamma_{2}-\epsilon / 2\right)}=1$.

Set $S_{1}=(-\delta, \delta) \times\left(-L_{1} \delta-\tau, 0\right)$,

$$
\mu_{1}=\frac{\cos \left(\gamma_{1}-\epsilon / 2\right)}{\cos (\sigma+\epsilon / 2)} \quad \text { and } \quad \mu_{2}=\frac{\cos \left(\gamma_{2}-\epsilon / 2\right)}{\cos (\pi-2 \alpha-\sigma+\epsilon / 2)} .
$$

Then $\mu_{1}<1, \mu_{2}<1$ and

$$
\sqrt{1+\left(L_{1}^{+}\right)^{2}} \cos \left(\gamma \circ R_{1}^{-1}(x)\right) \leq \mu_{1} \quad \text { for } x \in R_{1}\left(\partial^{+} \Omega\right) \cap S_{1},
$$


and

$$
\sqrt{1+\left(L_{1}^{-}\right)^{2}} \cos \left(\gamma \circ R_{1}^{-1}(x)\right) \leq \mu_{2} \quad \text { for } x \in R_{1}\left(\partial^{-} \Omega\right) \cap S_{1},
$$

We will now establish

$$
\int_{\Sigma \times(-T, T)} \cos (\gamma) h^{*} d H_{2} \leq \mu \int_{\mathscr{A}_{\lambda} \times(-T, T)}|D h|+\Upsilon \int_{\mathscr{A}_{\lambda} \times(-T, T)} h,
$$

when $h \in C^{1}(\Omega \times(-T, T)) \cap B V(\Omega \times(-T, T))$ with $h \geq 0$ in $\left.\Omega \times(-T, T)\right)$. To a great extent, we will follow the proof of [Finn 1986, Lemma 6.1]. In Definition 3.4, set $\epsilon$ equal to $\delta, N=N(\delta), N_{1}=N_{1}(\delta), \tau=\tau(\delta)$ and obtain a finite, open cover $\left\{\Lambda_{j}^{\delta}: j=2, \ldots, N\right\}$ of $\Omega^{\delta}$ in $\mathbb{R}^{2}$ with the properties described in the definition. Set $\Omega_{j}^{\delta}=\Lambda_{j}^{\delta} \cap \bar{\Omega}$ for $j=2, \ldots, N$ and set $\Omega_{1}^{\delta}=R_{1}^{-1}\left(S_{1}\right) \cap \bar{\Omega}$. Notice that $\left\{\Omega_{j}^{\delta}: j=1, \ldots, N\right\}$ is an open (in the relative topology of $\bar{\Omega}$ ) cover of $\bar{\Omega}$. Let $\left\{\varphi_{j}: j=1, \ldots, N\right\}$ be a partition of unity of $\bar{\Omega}$ subordinate to $\left\{\Omega_{j}^{\delta}: j=1, \ldots, N\right\}$. Notice since $O \notin \bigcup_{j=2}^{N} \overline{\Lambda_{j}^{\delta}}$ that $\varphi_{1} \equiv 1$ in some neighborhood of $O$.

Using the techniques in the proof of [Finn 1986, Lemma 6.1], one sees that

$$
\left|\int_{\Sigma \times(-T, T)} \varphi_{j} \cos (\gamma) h^{*} d H_{2}\right| \leq a(\delta) \int_{\mathscr{A}_{\lambda} \times(-T, T)} \varphi_{j}|D h|+\Upsilon \int_{\mathscr{A}_{\lambda}^{j} \times(-T, T)} h,
$$

where $\mathscr{A}_{\lambda}^{j}=\mathscr{A}_{\lambda} \cap F_{j}^{-1}\left(T_{j}\right)$ for each $j=2, \ldots, N_{1}$ and $k \in \mathbb{N}$. Notice also that these techniques yield

$$
\left|\int_{\partial^{+} \Omega \times(-T, T)} \varphi_{1} h^{*} d H_{2}\right| \leq \sqrt{1+\left(L_{1}^{+}\right)^{2}} \int_{\mathscr{A}_{\lambda}^{+} \times(-T, T)} \varphi_{1}|D h|+\Upsilon \int_{\mathscr{A}_{\lambda}^{+} \times(-T, T)} h
$$

and

$$
\left|\int_{\partial^{-} \Omega \times(-T, T)} \varphi_{1} h^{*} d H_{2}\right| \leq \sqrt{1+\left(L_{1}^{-}\right)^{2}} \int_{\mathscr{A}_{\lambda}^{-} \times(-T, T)} \varphi_{1}|D h|+\Upsilon \int_{\mathscr{A}_{\lambda}^{-} \times(-T, T)} h,
$$

where

$$
\begin{aligned}
& \mathscr{A}_{\lambda}^{+}=\Omega \cap R_{1}^{-1}\left(\left\{(x, y): 0<x<\delta, \psi_{1}^{+}(x)-\lambda<y<\psi_{1}^{+}(x)\right\}\right), \\
& \mathscr{A}_{\lambda}^{-}=\Omega \cap R_{1}^{-1}\left(\left\{(x, y):-\delta<x<0, \psi_{1}^{-}(x)-\lambda<y<\psi_{1}^{-}(x)\right\}\right) .
\end{aligned}
$$

Then

$$
\begin{aligned}
\int_{\Sigma \times(-T, T)} \varphi_{1} \cos (\gamma) h^{*} d H_{2} \leq \mu_{1} \int_{\mathscr{A}_{\lambda}^{+} \times(-T, T)} & \varphi_{1}|D h|+\Upsilon \int_{\mathscr{A}_{\lambda}^{+} \times(-T, T)} h \\
& +\mu_{2} \int_{\mathscr{A}_{\lambda}^{-} \times(-T, T)} \varphi_{1}|D h|+\Upsilon \int_{\mathscr{A}_{\lambda}^{-} \times(-T, T)} h,
\end{aligned}
$$


and so, if we set $\mu_{0}=\max \left\{\mu_{1}, \mu_{2}\right\}<1$,

$$
\int_{\Sigma \times(-T, T)} \varphi_{1} \cos (\gamma) h^{*} d H_{2} \leq \mu_{0} \int_{\mathscr{A}_{\lambda} \times(-T, T)} \varphi_{1}|D h|+\Upsilon \int_{\mathscr{A}_{\lambda}^{1} \times(-T, T)} h,
$$

where $\mathscr{A}_{\lambda}^{1}=\mathscr{A}_{\lambda} \cap B_{2 \zeta}(O)$. Therefore, if we set $\mu=\max \left\{a(\delta), \mu_{0}\right\}<1$, we obtain

$$
\begin{aligned}
\int_{\Sigma \times(-T, T)} \cos (\gamma) h^{*} d H_{2} & =\sum_{j=1}^{N_{1}} \int_{\Sigma \times(-T, T)} \varphi_{j} \cos (\gamma) h^{*} d H_{2} \\
& \leq \sum_{j=1}^{N_{1}}\left(\mu \int_{\mathscr{A}_{\lambda} \times(-T, T)} \varphi_{j}|D h|+\Upsilon \int_{\mathscr{A}_{\lambda}^{j} \times(-T, T)} h\right) \\
& \leq \mu \int_{\mathscr{A}_{\lambda} \times(-T, T)}|D h|+\Upsilon_{1} \int_{\mathscr{A}_{\lambda} \times(-T, T)} h
\end{aligned}
$$

and thus we obtain (56).

Now set $h=f_{k}$ and obtain $\int_{\Sigma} \cos (\gamma) f_{k}^{*} d s \leq \mu \int_{\mathscr{A}_{\delta}}\left|D f_{k}\right|+\Upsilon_{1} \int_{\mathscr{A}_{\delta}}\left|f_{k}\right|$ for each $k \in \mathbb{N}$. From (50), (53) and [Giusti 1984, Proposition 1.13], we see that

$$
\lim _{k \rightarrow \infty} \int_{\mathscr{A}_{\lambda} \times(-T, T)}\left|D f_{k}\right| d x=\int_{\mathscr{A}_{\lambda} \times(-T, T)}|D f|
$$

and therefore using this together with (49) and (51) yields

$$
\begin{aligned}
\int_{\Sigma} \cos (\gamma) f^{*} d H^{2} & =\int_{\Sigma} \cos (\gamma) f_{k}^{*} d H^{2} \leq \mu \int_{\mathscr{A}_{\delta}}\left|D f_{k}\right|+\Upsilon_{1} \int_{\mathscr{A}_{\delta}}\left|f_{k}\right| \\
& \leq \mu \int_{\mathscr{A}_{\delta}}\left|D f_{k}\right|+\Upsilon_{1} \int_{\mathscr{A}_{\delta}}|f|+\Upsilon_{1} \int_{\mathscr{A}_{\delta}}\left|f-f_{k}\right| .
\end{aligned}
$$

If we take the limit as $k \rightarrow \infty$, we obtain

$$
\int_{\Sigma} \cos (\gamma) f^{*} d H^{2} \leq \mu \int_{\mathscr{A}_{\delta}}|D f|+\Upsilon_{1} \int_{\mathscr{A}_{\delta}}|f| \text {. }
$$

We wish to prove (58) with $f$ replaced by $-f$. Fix $\epsilon \in\left(0, \min \left\{\gamma_{1}, \pi / 2-\gamma_{1}\right\}\right)$. Let $\zeta \in\left(0, \delta^{*} / 2\right)$ be small enough that

(a) $\left|\gamma(x)-\gamma_{1}\right|<\epsilon / 2$ whenever $x \in \partial^{+} \Omega \backslash\{O\}$ with $|x| \leq 2 \zeta$, and

(b) $\left|\gamma(x)-\gamma_{2}\right|<\epsilon / 2$ whenever $x \in \partial^{-} \Omega \backslash\{O\}$ with $|x| \leq 2 \zeta$.

Notice that if $x \in \partial^{+} \Omega \backslash\{O\} \cap B_{2 \zeta}(O)$, then $\pi-\gamma(x)>\pi-\gamma_{1}-\epsilon>\pi / 2$ and so

$$
\cos (\pi-\gamma)<0 \quad \text { on }\left(\partial^{+} \Omega \backslash\{O\}\right) \cap B_{2 \zeta}(O) .
$$

Also, if $x \in \partial^{-} \Omega \backslash\{O\} \cap B_{2 \zeta}(O)$, then

$$
\left|\tau^{-}(x)+\alpha\right|<\epsilon / 2 \text { and } \pi-\gamma(x)>\pi-\gamma_{2}-\epsilon / 2>\pi / 2-\epsilon / 2 .
$$


Let $\tau=\zeta$ and let $R_{2}: \mathbb{R}^{2} \rightarrow \mathbb{R}^{2}$ be the rotation about the origin through the angle $\alpha-\pi$. Then $R_{2}\left(\partial^{-} \Omega\right) \cap B_{2 \zeta}(O)$ is the graph $y=\psi_{2}^{-}(x)$ of a Lipschitz function with Lipschitz constant $L_{2}^{-} \leq \tan (\epsilon / 2)$; notice that $\operatorname{dom}\left(\psi_{1}^{-}\right)=\left(x_{0}^{-}, 0\right]$, where $\left|\left(x_{0}^{-}, \psi_{1}^{-}\left(x_{0}^{-}\right)\right)\right|=2 \zeta$. Set $L_{2}=L_{2}^{-}$and let $\delta>0$ satisfy $\delta^{2}+\left(L_{2} \delta+\tau\right)^{2}=4 \zeta^{2}$ (so that $\left.\delta=\zeta\left(\left(3 L_{2}^{2}+4\right)^{1 / 2}-1\right) /\left(L_{2}^{2}+1\right)\right)$. For $-\delta \leq x<0$, we have $\pi-\gamma(x) \geq$ $\pi / 2-\epsilon / 2$ and so

$$
\cos (\pi-\gamma(x)) \sqrt{1+\left(L_{2}^{-}\right)^{2}}<\cos (\pi / 2-\epsilon / 2) \sec (\epsilon / 2)<\frac{\cos \left(\pi / 4+\gamma_{1} / 2\right)}{\cos \left(\pi / 4-\gamma_{1} / 2\right)}<1 .
$$

We will now establish

$$
-\int_{\Sigma \times(-T, T)} \cos (\gamma) h^{*} d H_{2} \leq \mu \int_{\mathscr{A}_{\lambda} \times(-T, T)}|D h|+\Upsilon_{1} \int_{\mathscr{A}_{\lambda} \times(-T, T)} h
$$

when $h \in C^{1}(\Omega \times(-T, T)) \cap B V(\Omega \times(-T, T))$ with $h \geq 0$ in $\left.\Omega \times(-T, T)\right)$, where $\mu=\max \left\{a(\delta), \mu_{3}\right\}<1$ and $\mu_{3}=\cos (\pi / 2-\epsilon / 2) / \cos (\epsilon / 2)$. Let us write (60) as

$$
\int_{\Sigma \times(-T, T)} \cos (\pi-\gamma) h^{*} d H_{2} \leq \mu \int_{\mathscr{A}_{\lambda} \times(-T, T)}|D h|+\Upsilon_{1} \int_{\mathscr{A}_{\lambda} \times(-T, T)} h .
$$

Using the techniques in the proof of [Finn 1986, Lemma 6.1], one sees that

$$
\left|\int_{\Sigma \times(-T, T)} \varphi_{j} \cos (\pi-\gamma) h^{*} d H_{2}\right| \leq a(\delta) \int_{\mathscr{A}_{\lambda} \times(-T, T)} \varphi_{j}|D h|+\Upsilon \int_{\mathscr{A}_{\lambda}^{j} \times(-T, T)} h,
$$

where $\mathscr{A}_{\lambda}^{j}=\mathscr{A}_{\lambda} \cap F_{j}^{-1}\left(T_{j}\right)$ for each $j=2, \ldots, N_{1}$ and $k \in \mathbb{N}$. Notice also that these techniques yield

$$
\left|\int_{\partial^{-} \Omega \times(-T, T)} \varphi_{1} h^{*} d H_{2}\right| \leq \sqrt{1+\left(L_{1}^{-}\right)^{2}} \int_{\mathscr{A}_{\lambda}^{-} \times(-T, T)} \varphi_{1}|D h|+\Upsilon \int_{\mathscr{A}_{\lambda}^{-} \times(-T, T)} h,
$$

where $\mathscr{A}_{\lambda}^{-}=\Omega \cap R_{2}^{-1}\left(\left\{(x, y):-\delta<x<0, \psi_{2}^{-}(x)-\lambda<y<\psi_{2}^{-}(x)\right\}\right)$. Then

(61) $\int_{\Sigma \times(-T, T)} \varphi_{1} \cos (\pi-\gamma) h^{*} d H_{2} \leq \mu_{3} \int_{\mathscr{A}_{\lambda}^{-} \times(-T, T)} \varphi_{1}|D h|+\Upsilon \int_{\mathscr{A}_{\lambda}^{-} \times(-T, T)} h$.

Therefore, if we set $\mu=\max \left\{a(\delta), \mu_{0}\right\}<1$, we obtain

$$
\begin{aligned}
\int_{\Sigma \times(-T, T)} \cos (\pi-\gamma) h^{*} d H_{2} & =\sum_{j=1}^{N_{1}} \int_{\Sigma \times(-T, T)} \varphi_{j} \cos (\gamma) h^{*} d H_{2} \\
& \leq \sum_{j=1}^{N_{1}}\left[\mu \int_{\mathscr{A}_{\lambda} \times(-T, T)} \varphi_{j}|D h|+\Upsilon \int_{\mathscr{A}_{\lambda}^{j} \times(-T, T)} h\right] \\
& \leq \mu \int_{\mathscr{A}_{\lambda} \times(-T, T)}|D h|+\Upsilon_{1} \int_{\mathscr{A}_{\lambda} \times(-T, T)} h,
\end{aligned}
$$


and thus we obtain (60). If we reason as before when we used (56) to obtain (58), we see that (60) and the approximation of $f$ by the $\left(f_{k}\right)$ implies

$$
-\int_{\Sigma} \cos (\gamma) f^{*} d H^{2} \leq \mu \int_{\mathscr{A}_{\delta}}|D f|+\Upsilon_{1} \int_{\mathscr{A}_{\delta}}|f| .
$$

Since (58) and (62) are together equivalent to (48), we see that the lemma is proven when $\left(\gamma_{1}, \gamma_{2}\right) \in D_{2}^{+}$and $\gamma_{2} \leq \pi / 2$.

Case $2\left(\left(\gamma_{1}, \gamma_{2}\right) \in D_{2}^{+}\right.$and $\left.\gamma_{1} \geq \pi / 2\right)$. In this case, $\gamma_{2}>\pi / 2$. Let us set $\tilde{\gamma}=\pi-\gamma$, $\tilde{\gamma}_{1}=\pi-\gamma_{1}$ and $\tilde{\gamma}_{2}=\pi-\gamma_{2}$. Notice that $\tilde{\gamma}_{1}-\tilde{\gamma}_{2}=\gamma_{2}-\gamma_{1}>\pi-2 \alpha$ and so $\left(\tilde{\gamma}_{1}, \tilde{\gamma}_{2}\right) \in D_{2}^{-}$. Then $\left(\tilde{\gamma}_{2}, \tilde{\gamma}_{1}\right) \in D_{2}^{+}$with $\tilde{\gamma}_{1} \leq \pi / 2$. By reflecting $\Omega$ and $\gamma$ about the $x$-axis, we see from our previous argument with $\gamma_{2} \leq \pi / 2$ that (48) holds.

Case $3\left(\left(\gamma_{1}, \gamma_{2}\right) \in D_{2}^{+}, \quad \gamma_{1}<\pi / 2\right.$ and $\left.\gamma_{2}>\pi / 2\right)$. We use the same argument used to establish (48) when $\gamma_{2} \leq \pi / 2$ - that is, only one of the sides, $\partial^{+} \Omega$ or $\partial^{-} \Omega$, contributes to each integral since an inequality like (59) holds on the other side, and, by rotating through a suitable angle, we can make the intersection of the contributing side with a sufficiently small ball centered at $O$ the graph of a function over the $x$-axis with arbitrarily small Lipschitz constant. Then we see that (48) holds in this case.

Case $4\left(\left(\gamma_{1}, \gamma_{2}\right) \in D_{2}^{-}\right)$. In this case $\gamma_{1}-\gamma_{2}>\pi-2 \alpha$. Then $\left(\gamma_{2}, \gamma_{1}\right) \in D_{2}^{+}$and, by reflection about the $x$-axis, we see our previous arguments show that (48) holds.

Remark 3.2. Suppose $\Omega_{j} \rightarrow \Omega_{\infty}$ in that $\Omega_{j}=\left\{x \in \mathbb{R}^{2}: \epsilon_{j} x \in \Omega\right\}$ when $\epsilon_{j} \rightarrow 0$ as $j \rightarrow \infty$ and $\Omega_{\infty}=\{(r \cos (\theta), r \sin (\theta)): r>0,-\alpha<\theta<\alpha\}$. Assuming we define other quantities appropriately (for example, $\gamma_{j} \in C^{0}\left(\partial \Omega_{j}\right)$ defined by $\gamma_{j}(x)=\gamma\left(\epsilon_{j} x\right)$ for $\left.x \in \partial \Omega_{j}\right)$, then an examination of the proofs of [Finn 1986, Lemmas 6.1 and 7.6] shows that the constants $\zeta, a(\zeta), \Upsilon$ and $\mu$ can be assumed to be independent of $j$ in Lemma 3.1.

Remark 3.3. Notice, in particular, that if $U$ is a Caccioppoli set in $\Omega \times \mathbb{R}$, then, with $f=\phi_{U}$ and $f=\phi_{U^{\prime}}$, (48) implies

$$
\left|\int_{\Sigma \times(-T, T)} \cos (\gamma) \phi_{U}^{*} d H_{2}\right| \leq \mu \int_{\Sigma_{\lambda} \times(-T, T)}\left|D \phi_{U}\right|+\Upsilon \int_{\Sigma_{\lambda} \times(-T, T)} \phi_{U}
$$

and

$$
\left|\int_{\Sigma \times(-T, T)} \cos (\pi-\gamma) \phi_{U^{\prime}}^{*} d H_{2}\right| \leq \mu \int_{\Sigma_{\lambda \times(-T, T)}}\left|D \phi_{U^{\prime}}\right|+\Upsilon \int_{\Sigma_{\lambda \times(-T, T)}} \phi_{U^{\prime}},
$$

where $U^{\prime}=(\Omega \times \mathbb{R}) \backslash U$ for $T>0$.

Emmer's lemma (for example, [Emmer 1973]), in this case Lemma 3.1, is the key ingredient needed to obtain lower semicontinuity of the functional in question. Slight modifications of arguments in [Finn 1986, Section 7.4] and [Tam 1986b, 
Lemma 1.2] show that $\mathscr{E}$ and $\mathscr{F}_{T, K}$ for $T>0$ and $K \subset \subset \mathbb{R}^{2}$ are lower semicontinuous. The proof of [Tam 1986a, Lemma 2.3] (also [Tam 1984, Lemma 2.3]), adapted to the situation here, yields this:

Lemma 3.2. Let $\Omega$ and $\gamma$ be as in Theorem 1.1, and note that $(\gamma, \Omega, O)$ is admissible. Let $\left(\epsilon_{j}\right)$ be a sequence of positive reals such that $\lim _{j \rightarrow \infty} \epsilon_{j}=0$. For each $j \in \mathbb{N}$, set $H_{j}^{*}(x)=\epsilon_{j} H^{*}\left(\epsilon_{j} x\right)$ for $x \in \Omega_{j}$ and $\gamma_{j}(x)=\epsilon_{j} \gamma\left(\epsilon_{j} x\right)$ for $x \in \partial \Omega_{j}$. For each $j \in \mathbb{N}$, suppose $f_{j}$ is a generalized solution for

$$
\mathscr{E}_{j}(u)=\int_{\Omega_{j}} \sqrt{1+|D u|^{2}} d H_{2}+\int_{\Omega_{j}} H_{j}^{*} u d H_{2}-\int_{\partial \Omega_{j}} \cos (\gamma) u d H_{1} .
$$

Then $\left(f_{j}\right)$ has a subsequence $\left(f_{j_{i}}\right)$ that converges locally to a generalized solution $f_{\infty}$ for

$$
\mathscr{E}_{\infty}(u)=\int_{\Omega_{\infty}} \sqrt{1+|D u|^{2}} d H_{2}-\int_{\partial^{+} \Omega_{\infty}} \cos \left(\gamma_{1}\right) u d H_{1}-\int_{\partial^{-} \Omega_{\infty}} \cos \left(\gamma_{2}\right) u d H_{1} .
$$

\section{Acknowledgments}

I wish to thank Harold Parks for his comments and Leon Simon for his comments and his sketch of an alternative proof of [Simon 1980, Section 4] using a scaling argument (for example, [Simon 1976]). I thank the referee for efforts and suggestions. I especially thank Robert Finn for his support and encouragement over the years, his discovery and investigation (with Paul Concus and others) of this conjecture and his editorial efforts. I am indebted to Eberhard Zeidler and the Max-Planck-Institut für Mathematik in den Naturwissenschaften, to Robert Finn, Laurence Nédélec, Rafe Mazzeo, Leon Simon and the Department of Mathematics at Stanford University and to Ben Andrews, Maria Athanassenas and the Centre for Mathematics and its Applications of the Australian National University for their hospitality during some of the course of this study.

\section{References}

[Allard 1972] W. K. Allard, "On the first variation of a varifold", Ann. of Math. (2) 95 (1972), 417-491. MR 46 \#6136 Zbl 0252.49028

[Athanassenas and Lancaster 2008] M. Athanassenas and K. Lancaster, "CMC capillary surfaces at reentrant corners”, Pacific J. Math. 234:2 (2008), 201-228. MR 2009b:53007 Zbl 1148.76010

[Bear and Hile 1983] H. S. Bear and G. N. Hile, "Behavior of solutions of elliptic differential inequalities near a point of discontinuous boundary data", Comm. Partial Differential Equations 8:11 (1983), 1175-1197. MR 85g:35054 Zbl 0533.35040

[Concus and Finn 1996] P. Concus and R. Finn, "Capillary wedges revisited", SIAM J. Math. Anal. 27:1 (1996), 56-69. MR 96m:76006 Zbl 0843.76012

[Concus et al. 1992] P. Concus, R. Finn, and F. Zabihi, "On canonical cylinder sections for accurate determination of contact angle in microgravity", pp. 125-131 in Fluid Mechanics Phenomena in 
Microgravity, Applied Mechanics Division 154, American Society of Mechanical Engineers, New York, 1992.

[Courant 1977] R. Courant, Dirichlet's principle, conformal mapping, and minimal surfaces, Springer, New York, 1977. MR 56 \#13103 Zbl 0354.30012

[Elcrat and Lancaster 1986] A. R. Elcrat and K. E. Lancaster, "Boundary behavior of a nonparametric surface of prescribed mean curvature near a reentrant corner", Trans. Amer. Math. Soc. 297:2 (1986), 645-650. MR 87h:35098 Zbl 0602.35042

[Emmer 1973] M. Emmer, "Esistenza, unicità e regolarità nelle superfici de equilibrio nei capillari”, Ann. Univ. Ferrara Sez. VII (N.S.) 18 (1973), 79-94. MR 49 \#1281 Zbl 0275.49005

[Federer 1969] H. Federer, Geometric measure theory, Die Grundlehren der mathematischen Wissenschaften 153, Springer, New York, 1969. MR 41 \#1976 Zbl 0176.00801

[Finn 1986] R. Finn, Equilibrium capillary surfaces, Die Grundlehren der Mathematischen Wissenschaften 284, Springer, New York, 1986. MR 88f:49001 Zbl 0583.35002

[Finn 1988a] R. Finn, "Comparison principles in capillarity", pp. 156-197 in Partial differential equations and calculus of variations, edited by S. Hildebrandt and R. Leis, Lecture Notes in Math. 1357, Springer, Berlin, 1988. MR 90d:53010 Zbl 0692.35006

[Finn 1988b] R. Finn, "Moon surfaces, and boundary behaviour of capillary surfaces for perfect wetting and nonwetting", Proc. London Math. Soc. (3) 57:3 (1988), 542-576. MR 89m:49076 Zbl 0668.76019

[Finn 1996] R. Finn, "Local and global existence criteria for capillary surfaces in wedges", Calc. Var. Partial Differential Equations 4:4 (1996), 305-322. MR 97f:53005 Zbl 0872.76017

[Finn 1999] R. Finn, “Capillary surface interfaces”, Notices Amer. Math. Soc. 46:7 (1999), 770-781. MR 2000g:76033

[Finn 2002a] R. Finn, "Eight remarkable properties of capillary surfaces", Math. Intelligencer 24:3 (2002), 21-33. MR 2003f:76041

[Finn 2002b] R. Finn, "Some properties of capillary surfaces", Milan J. Math. 70 (2002), 1-23. MR 2003m:53012 Zbl 1053.76009

[Finn and Serrin 1958] R. Finn and J. Serrin, "On the Hölder continuity of quasi-conformal and elliptic mappings", Trans. Amer. Math. Soc. 89 (1958), 1-15. MR 20 \#4094 Zbl 0082.29401

[Gehring 1973] F. W. Gehring, "The $L^{p}$-integrability of the partial derivatives of a quasiconformal mapping”, Acta Math. 130 (1973), 265-277. MR 53 \#5861 Zbl 0258.30021

[Gehring 2005] F. W. Gehring, "Quasiconformal mappings in Euclidean spaces", pp. 1-29 in Handbook of complex analysis: Geometric function theory, vol. 2, edited by R. Kühnau, Elsevier, Amsterdam, 2005. MR 2005k:30044 Zbl 1078.30014

[Gilbarg and Trudinger 1983] D. Gilbarg and N. S. Trudinger, Elliptic partial differential equations of second order, 2nd ed., Grundlehren der Mathematischen Wissenschaften 224, Springer, Berlin, 1983. MR 86c:35035 Zbl 0562.35001

[Giusti 1980] E. Giusti, "Generalized solutions for the mean curvature equation", Pacific J. Math. 88:2 (1980), 297-321. MR 83a:35030 Zbl 0461.49024

[Giusti 1984] E. Giusti, Minimal surfaces and functions of bounded variation, Monographs in Mathematics 80, Birkhäuser, Basel, 1984. MR 87a:58041 Zbl 0545.49018

[Iwaniec and Martin 2001] T. Iwaniec and G. Martin, Geometric function theory and non-linear analysis, Oxford University Press, New York, 2001. MR 2003c:30001 Zbl 1045.30011

[Jeffres and Lancaster 2007] T. Jeffres and K. Lancaster, "Vertical blow ups of capillary surfaces in $\mathbb{R}^{3}$, I: Convex corners", Electron. J. Differential Equations (2007), no. 152. MR 2009b:49103

[Kenmotsu 1979] K. Kenmotsu, "Weierstrass formula for surfaces of prescribed mean curvature", Math. Ann. 245:2 (1979), 89-99. MR 81c:53005b Zbl 0402.53002 
[Kenmotsu 2003] K. Kenmotsu, Surfaces with constant mean curvature, Translations of Mathematical Monographs 221, American Mathematical Society, Providence, RI, 2003. MR 2004m:53014 Zbl 1042.53001

[Lancaster 1985] K. E. Lancaster, "Boundary behavior of a nonparametric minimal surface in $\mathbf{R}^{3}$ at a nonconvex point", Analysis 5:1-2 (1985), 61-69. Corrected in 6 (1986), 413. MR 86m:49053 Zbl 0601.35035

[Lancaster and Siegel 1996a] K. E. Lancaster and D. Siegel, "Existence and behavior of the radial limits of a bounded capillary surface at a corner", Pacific J. Math. 176:1 (1996), 165-194. Figures corrected in 179:2 (1997), 397-402. MR 98g:58030a Zbl 0866.76018

[Lancaster and Siegel 1996b] K. E. Lancaster and D. Siegel, "Behavior of a bounded non-parametric $H$-surface near a reentrant corner”, Z. Anal. Anwendungen 15:4 (1996), 819-850. MR 97m:53011 Zbl 0866.35046

[Massari and Pepe 1975] U. Massari and L. Pepe, "Successioni convergenti di ipersuperfici di curvatura media assegnata", Rend. Sem Mat. Univ. Padova 53 (1975), 53-68. MR 54 \#8415 Zbl 0358.49020

[Miranda 1977] M. Miranda, "Superficie minime illimitate", Ann. Scuola Norm. Sup. Pisa Cl. Sci. (4) 4:2 (1977), 313-322. MR 58 \#18063 Zbl 0352.49020

[Monakhov 1983] V. N. Monakhov, Boundary value problems with free boundaries for elliptic systems of equations, Translations of Mathematical Monographs 57, American Mathematical Society, Providence, RI, 1983. MR 85a:35029 Zbl 0532.35001

[Shi and Finn 2004] D. Shi and R. Finn, "On a theorem of Lancaster and Siegel", Pacific J. Math. 213:1 (2004), 111-119. MR 2004m:76038 Zbl 1156.76361

[Simon 1976] L. Simon, "Remarks on curvature estimates for minimal hypersurfaces", Duke Math. J. 43:3 (1976), 545-553. MR 54 \#6040 Zbl 0348.53003

[Simon 1980] L. Simon, "Regularity of capillary surfaces over domains with corners", Pacific J. Math. 88:2 (1980), 363-377. MR 82d:49043 Zbl 0467.35022

[Tam 1984] L. F. Tam, The Behavior of Capillary Surfaces as Gravity Tends to Zero, thesis, Stanford University, 1984.

[Tam 1986a] L.-F. Tam, “The behavior of capillary surfaces as gravity tends to zero", Comm. Partial Differential Equations 11:8 (1986), 851-901. MR 89c:76040 Zbl 0607.35041

[Tam 1986b] L.-F. Tam, "On existence criteria for capillary free surfaces without gravity”, Pacific J. Math. 125:2 (1986), 469-485. MR 88a:49023 Zbl 0604.49030

[Tam 1986c] L.-F. Tam, "Regularity of capillary surfaces over domains with corners: Borderline case”, Pacific J. Math. 124:2 (1986), 469-482. MR 87k:49049 Zbl 0604.49029

[Taylor 1976] J. E. Taylor, "The structure of singularities in soap-bubble-like and soap-film-like minimal surfaces", Ann. of Math. (2) 103:3 (1976), 489-539. MR 55 \#1208a Zbl 0335.49032

[Taylor 1977] J. E. Taylor, "Boundary regularity for solutions to various capillarity and free boundary problems", Comm. Partial Differential Equations 2:4 (1977), 323-357. MR 58 \#7336 Zbl 0357.35010

Received December 28, 2007. Revised March 17, 2010.

\section{KIRK E. LANCASTER}

DEPARTMENT OF MATHEMATICS AND STATISTICS

WICHITA STATE UNIVERSITY

WICHITA, KS 67260-0033

UNITED STATES

lancaster@math.wichita.edu 


\title{
PACIFIC JOURNAL OF MATHEMATICS
}

\author{
http://www.pjmath.org \\ Founded in 1951 by \\ E. F. Beckenbach (1906-1982) and F. Wolf (1904-1989)
}

\section{EDITORS}

V. S. Varadarajan (Managing Editor)

Department of Mathematics

University of California

Los Angeles, CA 90095-1555

pacific@math.ucla.edu

Vyjayanthi Chari

Department of Mathematics

University of California

Riverside, CA 92521-0135

chari@math.ucr.edu

Robert Finn

Department of Mathematics Stanford University

Stanford, CA 94305-2125

finn@math.stanford.edu

Kefeng Liu

Department of Mathematics

University of California

Los Angeles, CA 90095-1555

liu@math.ucla.edu
Darren Long

Department of Mathematics

University of California

Santa Barbara, CA 93106-3080

long@math.ucsb.edu

Jiang-Hua Lu

Department of Mathematics

The University of Hong Kong

Pokfulam Rd., Hong Kong jhlu@maths.hku.hk

Alexander Merkurjev

Department of Mathematics

University of California

Los Angeles, CA 90095-1555

merkurev@math.ucla.edu
Sorin Popa

Department of Mathematics University of California

Los Angeles, CA 90095-1555 popa@math.ucla.edu

Jie Qing

Department of Mathematics

University of California

Santa Cruz, CA 95064

qing@cats.ucsc.edu

Jonathan Rogawski

Department of Mathematics

University of California

Los Angeles, CA 90095-1555

jonr@math.ucla.edu

\section{PRODUCTION}

pacific@math.berkeley.edu

\begin{abstract}
Silvio Levy, Scientific Editor Matthew Cargo, Senior Production Editor
\end{abstract}
ACADEMIA SINICA, TAIPEI

CALIFORNIA INST. OF TECHNOLOGY

INST. DE MATEMÁTICA PURA E APLICADA

KEIO UNIVERSITY

MATH. SCIENCES RESEARCH INSTITUTE

NEW MEXICO STATE UNIV.

OREGON STATE UNIV.

\section{SUPPORTING INSTITUTIONS}

STANFORD UNIVERSITY
UNIV. OF BRITISH COLUMBIA
UNIV. OF CALIFORNIA, BERKELEY
UNIV. OF CALIFORNIA, DAVIS
UNIV. OF CALIFORNIA, LOS ANGELES
UNIV. OF CALIFORNIA, RIVERSIDE
UNIV. OF CALIFORNIA, SAN DIEGO
UNIV. OF CALIF., SANTA BARBARA

UNIV. OF CALIF., SANTA CRUZ

UNIV. OF MONTANA

UNIV. OF OREGON

UNIV. OF SOUTHERN CALIFORNIA

UNIV. OF UTAH

UNIV. OF WASHINGTON

WASHINGTON STATE UNIVERSITY

These supporting institutions contribute to the cost of publication of this Journal, but they are not owners or publishers and have no responsibility for its contents or policies.

See inside back cover or www.pjmath.org for submission instructions.

The subscription price for 2010 is US \$420/year for the electronic version, and \$485/year for print and electronic.

Subscriptions, requests for back issues from the last three years and changes of subscribers address should be sent to Pacific Journal of Mathematics, P.O. Box 4163, Berkeley, CA 94704-0163, U.S.A. Prior back issues are obtainable from Periodicals Service Company, 11 Main Street, Germantown, NY 12526-5635. The Pacific Journal of Mathematics is indexed by Mathematical Reviews, Zentralblatt MATH, PASCAL CNRS Index, Referativnyi Zhurnal, Current Mathematical Publications and the Science Citation Index.

The Pacific Journal of Mathematics (ISSN 0030-8730) at the University of California, c/o Department of Mathematics, 969 Evans Hall, Berkeley, CA 94720-3840, is published monthly except July and August. Periodical rate postage paid at Berkeley, CA 94704, and additional mailing offices. POSTMASTER: send address changes to Pacific Journal of Mathematics, P.O. Box 4163, Berkeley, CA 94704-0163.

PJM peer review and production are managed by EditFLOW ${ }^{\mathrm{TM}}$ from Mathematical Sciences Publishers.

PUBLISHED BY PACIFIC JOURNAL OF MATHEMATICS

at the University of California, Berkeley 94720-3840

A NON-PROFIT CORPORATION

Typeset in LATEX

Copyright $(\mathrm{C} 2010$ by Pacific Journal of Mathematics 


\section{PACIFIC JOURNAL OF MATHEMATICS}

Volume $247 \quad$ No. $1 \quad$ September 2010

Classification results for easy quantum groups

TEOdor BANiCA, StePhen CURRAN and Roland SPEICHER

Batalin-Vilkovisky coalgebra of string topology

XiAOJUn CHEN and WeE LiAnG GAN

Invariant Finsler metrics on polar homogeneous spaces

SHAOQIANG DENG

A proof of the Concus-Finn conjecture

KIRK E. LANCASTER

The existence and monotonicity of a three-dimensional transonic shock in a finite nozzle with axisymmetric exit pressure

JUn Li, ZHOUPING XIN and HUICHENG YiN

Bi-Hamiltonian flows and their realizations as curves in real semisimple homogeneous manifolds

GLORIA MARÍ BEFFA

Closed orbits of a charge in a weakly exact magnetic field

WILL J. MERRY

Ringel-Hall algebras and two-parameter quantized enveloping algebras

XIN TANG

A new probability distribution with applications

MINGJIN WANG 